\title{
Chemical evolution in the environment of intermediate mass young stellar objects ${ }^{\star}$ NGC 7129 - FIRS 2 and LkH $\alpha 234$
}

\author{
A. Fuente ${ }^{1}$, J. R. Rizzo ${ }^{1,2}$, P. Caselli ${ }^{3}$, R. Bachiller ${ }^{1}$, and C. Henkel ${ }^{4}$ \\ 1 Observatorio Astronómico Nacional (IGN), Campus Universitario, Apdo. 112, 28803 Alcalá de Henares (Madrid), Spain \\ e-mail: a.fuente@oan.es \\ ${ }^{2}$ Departamento de Física, Universidad Europea de Madrid, Urb. El Bosque, 28670 Villaviciosa de Odón, Spain \\ 3 Osservatorio Astrofisico di Arcetri, Largo Enrico Fermi 5, 50125 Firenze, Italy \\ ${ }^{4}$ Max-Planck-Institut für Radioastronomie, Auf dem Hügel 69, 53121 Bonn, Germany
}

Received 27 August 2004 / Accepted 15 November 2004

\begin{abstract}
We have carried out a molecular survey of the Class 0 IM protostar NGC 7129 - FIRS 2 (hereafter FIRS 2) and the Herbig Be star $\mathrm{LkH} \alpha 234$ with the aim of studying the chemical evolution of the envelopes of intermediate-mass (IM) young stellar objects (YSOs). The two objects have similar luminosities $\left(\sim 500 L_{\odot}\right)$ and are located in the same molecular cloud which minimizes the chemical differences due to different stellar masses or initial cloud conditions. Moreover, since they are located at the same distance, we have the same spatial resolution in both objects. A total of 17 molecular species (including rare isotopes) have been observed in both objects and the structure of their envelopes and outflows has been determined with unprecedent detail.

Our results show that the protostellar envelopes are dispersed and warmed up during the evolution of the YSO into a pre-main sequence star. In fact, the envelope mass decreases by a factor $>5$ from FIRS 2 to $\mathrm{LkH} \alpha 234$, while the kinetic temperature increases from $\sim 13 \mathrm{~K}$ to $28 \mathrm{~K}$. On the other hand, there is no molecular outflow associated with $\mathrm{LkH} \alpha 234$. The molecular outflow seems to stop before the star becomes visible.

These physical changes strongly affect the chemistry of their envelopes. $\mathrm{The}_{2} \mathrm{~N}_{2} \mathrm{H}^{+}$and $\mathrm{NH}_{3}$ abundances seem to be quite similar in the two objects. However, the $\mathrm{H}^{13} \mathrm{CO}^{+}$abundance is a factor of $\sim 3$ lower in the densest part of FIRS 2 than in LkH $\alpha 234$, very likely because of depletion. In contrast, the $\mathrm{SiO}$ abundance is larger by a factor of $\sim 100$ in FIRS 2 than in LkH $\alpha 234$. CS presents complex behavior since its emission arises in different envelope components (outflow, cold envelope, hot core) and could also suffer from depletion. The $\mathrm{CH}_{3} \mathrm{OH}$ and $\mathrm{H}_{2} \mathrm{CO}$ column densities are very similar in FIRS 2 and LkH $\alpha 234$ which implies that the beam-averaged abundances are a factor $>5$ larger in $\mathrm{LkH} \alpha 234$ than in FIRS 2. The same is found for the PDR tracers $\mathrm{CN}$ and $\mathrm{HCN}$ which have similar column densities in both objects. Finally, complex behavior is found for the deuterated compounds. While the $\mathrm{DCO}^{+} / \mathrm{H}^{13} \mathrm{CO}^{+}$ratio decreases by a factor of $\sim 4$ from FIRS 2 to $\mathrm{LkH} \alpha 234$, the $\mathrm{D}_{2} \mathrm{CO} / \mathrm{H}_{2} \mathrm{CO}$ ratios is within a factor $1.5 \mathrm{in}$ both objects. The detection of a warm $\mathrm{CH}_{3} \mathrm{CN}$ component with $T_{k}>63 \mathrm{~K}$ shows the existence of a hot core in FIRS 2. Thus far, only a handful of hot cores have been detected in low and intermediate mass stars.

Based on our results in FIRS 2 and $\mathrm{LkH} \alpha$ 234, we propose some abundance ratios that can be used as chemical clocks for the envelopes of IM YSOs. The $\mathrm{SiO} / \mathrm{CS}, \mathrm{CN} / \mathrm{N}_{2} \mathrm{H}^{+}, \mathrm{HCN} / \mathrm{N}_{2} \mathrm{H}^{+}, \mathrm{DCO}^{+} / \mathrm{HCO}^{+}$and $\mathrm{D}_{2} \mathrm{CO} / \mathrm{DCO}^{+}$ratios are good diagnostics of the protostellar evolutionary stage.
\end{abstract}

Key words. stars: formation - stars: pre-main sequence - stars: individual: $\mathrm{LkH} \alpha 234$ - ISM: abundances ISM: clouds - ISM: individual objects: NGC 7129

\section{Introduction}

Chemistry is a powerful tool for studying young stellar objects (YSOs) and their environments. On the one hand, chemistry is a diagnostic tool of the different envelope components. On the other hand, it is a good time indicator during the protostellar evolution. Chemical studies have been used to determine the

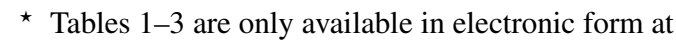
http://www . edpsciences.org physical structure of low-mass YSOs (e.g. Maret et al. 2004; Jørgensen et al. 2004a,b). These studies have revealed, for example, the presence of warm regions where the ices evaporate giving rise to regions similar to hot cores in massive protostars. However, the chemistry of the two classes of objects is different and raises questions on the mechanisms that lead to the observed chemical complexity and its dependence on the stellar mass (Cazaux et al. 2003; Bottinelli et al. 2004). 
Chemistry has also been used as a time evolution indicator in both low-mass and high-mass objects. For instance, Maret et al. (2004) studied a sample of low-mass Class 0 objects and found some indications that the $\mathrm{H}_{2} \mathrm{CO}$ abundance increases during the protostellar evolution. However, the different abundances can be due to different initial cloud conditions from which the protostars evolved. Further chemical studies are required to clarify this subject.

In this paper we present a chemical study of the two intermediate mass (IM) YSOs, FIRS 2 and $\mathrm{LkH} \alpha 234$. Contrary to low-mass protostars, IM YSOs have been very little studied thus far, especially young protostars. For example, out of 42 Class 0 sources compiled by André et al. (2000), only six had luminosities in excess of $40 L_{\odot}$ (the precursors of HAe stars) and only one had a luminosity of $\sim 10^{3} L_{\odot}$ (the precursor of a HBe star). IM YSOs $\left(M_{*} \sim 2-10 M_{\odot}\right)$ are not only an important link between low mass and high-mass stars but they share many characteristics of high mass star formation (clustering, PDR/HII regions) without the disadvantage of being too distant ( $d \lesssim 1 \mathrm{Kpc}$ ), or too complex. They are also important for the understanding of planet formation since Herbig Ae stars are the precursors of Vega-type systems. On a larger scale, they dominate the mean UV interstellar field in our Galaxy (Wolfire et al. 2003).

\section{Target selection and observational strategy}

FIRS 2 has been classified as a Class 0 IM object (Eiroa et al. 1998) and is, very likely, the youngest IM object known at present. An energetic bipolar molecular outflow is associated with it (Fuente et al. 2001). $\mathrm{LkH} \alpha 234$ is a embedded HBe star which still keeps a massive envelope $\left(M \sim 16 M_{\odot}\right)$ but no bipolar molecular outflow seems to be driven by it (Fuente et al. 2001, 2002). These objects have the peculiarity of having similar luminosities $\left(\sim 500 L_{\odot}\right)$ and be located in the same molecular cloud. During the protostellar and pre-main sequence evolution, the luminosity remains quite constant for a given stellar mass (André et al. 2000). Thus, the same luminosity implies similar stellar mass. In addition, both sources are located in the same molecular cloud. This minimizes chemical effects due to very different stellar mass (both of them have $M_{*} \sim 5 M_{\odot}$ ) and/or different initial cloud conditions. Thus, in spite of our reduced sample, we have an excellent opportunity of finding an evolutionary track for IM YSOs. In Fig. 1 we show the $1.3 \mathrm{~mm}$ continuum map of the reflection nebula NGC 7129 . Both FIRS 2 (NGC 7129-FIRS 2) and LkH $\alpha 234$ (NGC 7129 - FIRS 1) are associated with intense centrally peaked $1.3 \mathrm{~mm}$ continuum and far infrared sources. We have also overlaid the red and blue lobes (as traced by the ${ }^{12} \mathrm{CO} J=2 \rightarrow 1$ line) of the bipolar outflows associated to these YSOs (see Fuente et al. 2001, for a more detailed description). Clustering becomes significant in this range of stellar masses (Testi et al. 1999). Interferometric observations towards $\mathrm{LkH} \alpha 234$ show the existence of a young infrared companion (IRS 6) which is very likely the exciting source of the bipolar molecular outflow and the [SII] jet detected by Ray et al. (1990). The quadrupolar morphology of the outflow detected in NGC 7129-FIRS 2 is also due to the superposition of two bipolar molecular outflows

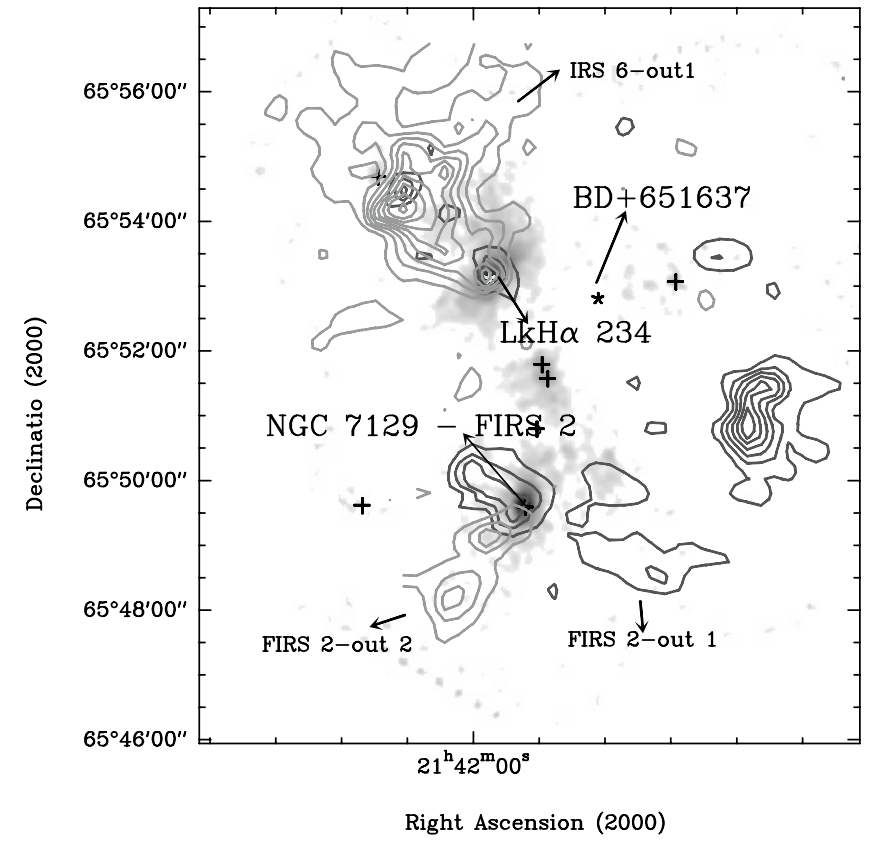

Fig. 1. Map of the $1.3 \mathrm{~mm}$ continuum flux toward NGC 7129 . Levels are $22.3,44.6,89.2,178.4$ to $713.6 \mathrm{mJy} /$ beam in steps of $178.4 \mathrm{mJy} / \mathrm{beam}$. Crosses indicate the millimeter sources and stars the infrared sources. The contours represent the redshifted and blueshifted high-velocity gas of the nebula as traced by the ${ }^{12} \mathrm{CO} J=2 \rightarrow 1$ line. Velocity intervals are $[-30,-13] \mathrm{km} \mathrm{s}^{-1}$ for the blue lobe (dark contours) and $[-7,11] \mathrm{km} \mathrm{s}^{-1}$ for the red-lobe (grey contours). Contours are 10 to $60 \mathrm{~K} \mathrm{~km} \mathrm{~s}^{-1}$ in steps of $10 \mathrm{~K} \mathrm{~km} \mathrm{~s}^{-1}$ for the blue lobe and 10 to $100 \mathrm{~K} \mathrm{~km} \mathrm{~s}^{-1}$ in steps of $15 \mathrm{~K} \mathrm{~km} \mathrm{~s}^{-1}$ for the red lobe.

FIRS 2-out 1 and FIRS 2-out 2 (Fuente et al. 2001). Fuente et al. (2001) proposed that FIRS 2-out 1 is associated with the Class 0 protostar while FIRS 2-out 2 is more likely associated with a more evolved infrared star (FIRS 2 - IR).

A complex chemical evolution occurs in the YSO envelopes during the protostellar evolution. This involves accretion of species in an icy mantle during the pre-collapse phase, followed by grain-surface chemistry and evaporation of ices once the YSO has started to heat its surroundings (e.g. Brown et al. 1988). In massive YSOs, the evaporated molecules drive a rapid high-temperature chemistry for a period of $10^{4}-10^{5}$ years, resulting in the complex, saturated organic molecules $\left(\mathrm{CH}_{3} \mathrm{OCH}_{3}, \mathrm{CH}_{3} \mathrm{CN}, \mathrm{C}_{2} \mathrm{H}_{5} \mathrm{OH}, \ldots\right)$ that are characteristic of a hot core (e.g. Charnley et al. 1992; Caselli et al. 1993; Rodgers \& Charnley 2003; Nomura \& Millar 2004; Viti et al. 2004). Once most of the envelope has been dispersed, the UV radiation can escape to form a photon-dominatedregion (PDR) and, in the case of massive stars, an HII region. Simultaneously, the energetic bipolar outflows develop a shock chemistry in the surrounding molecular cloud.

To discern between the different envelope components and determine the protostellar envelope evolution, we have selected a set of molecular tracers. Specifically, $\mathrm{NH}_{3}, \mathrm{~N}_{2} \mathrm{H}^{+}, \mathrm{H}^{13} \mathrm{CO}^{+}$ and $\mathrm{HC}^{18} \mathrm{O}^{+}$have been observed as tracers of the cold envelope; the volatile species $\mathrm{CH}_{3} \mathrm{OH}$ and $\mathrm{H}_{2} \mathrm{CO}$ to study the warm envelope where the icy grain mantles have evaporated; the complex molecule $\mathrm{CH}_{3} \mathrm{CN}$ to trace the hot core; and $\mathrm{CN}$ and $\mathrm{HCN}$ 
to trace the incipient $P D R$. In addition, we have also observed several $\mathrm{CS}$ and $\mathrm{C}^{34} \mathrm{~S}$ lines that are useful to constrain the physical conditions of the envelope, and $\mathrm{SiO}$ as an excellent tracer of the shock chemistry associated to the outflow. The observation of the deuterated compounds $\mathrm{N}_{2} \mathrm{D}^{+}$, $\mathrm{DCO}^{+}$and $\mathrm{D}_{2} \mathrm{CO}$ also provide some information about how the deuterium fractionation is affected by the YSO evolution. Obviously, the "molecular tracer" - "envelope component" correspondence is not unique and all the species have contributions from other envelope components. Arguments based on the morphology of the emission, kinematics and different excitation conditions are used to discern between the different components in these cases.

\section{Observations}

The $(J, K)=(1,1),(2,2),(3,3)$ and $(4,4)$ inversion lines of ammonia were observed using the Effelsberg 100-m radiotelescope of the MPIfR in December 2000 and October 2002. We observed only one position in both sources. The half power beam width (HPBW) of the telescope at the rest frequency of the $\mathrm{NH}_{3}$ lines, $23.7 \mathrm{GHz}$, was $40^{\prime \prime}$. We used the new cooled dual-channel HEMT $K$-band receiver with a typical system temperature of $200 \mathrm{~K}$ on a main beam brightness temperature scale. The 8192-channel autocorrelator was used as the backend. The four ammonia lines were observed simultaneously with a total bandwidth of $10 \mathrm{MHz}$ and a channel separation of $0.098 \mathrm{~km} \mathrm{~s}^{-1}$. We estimate that line intensities are accurate to within $\pm 15 \%$.

The main set of observations was carried out with the IRAM 30m telescope in Pico de Veleta (Spain) during three different observing periods in May 1999, July 2002 and August 2003. The list of observed lines, the telescope characteristics at each frequency and a summary of the observations are shown in Table 1. When possible, all the lines of the same molecule were observed simultaneously in order to avoid observational errors. The backends were an autocorrelator split in several parts and a $256 \times 100 \mathrm{kHz}$ filter-bank. All the lines were observed with a spectral resolution of $\sim 78 \mathrm{kHz}$ except in the cases that are explicitly indicated in Table 1 . The intensity scale used in this paper is main brightness temperature. Comparing the intensity of some pattern lines in different observing periods, we estimate that the calibration is accurate within $20 \%$ at $3 \mathrm{~mm}$ and within $30 \%$ at $1.3 \mathrm{~mm}$. We have observed high-S/Nratio spectra towards the star positions for all the lines listed in Table 1. In addition, we have obtained small maps in the most intense lines (see Table 1). In the case of the $\mathrm{SiO} 2 \rightarrow 1$, SiO $3 \rightarrow 2, \mathrm{H}^{13} \mathrm{CO}^{+} 1 \rightarrow 0$ and $\mathrm{CS} 3 \rightarrow 2$ lines we have mapped the entire outflows as traced by the high-velocity $\mathrm{CO}$ emission. Fits to the oberved lines at the $(0,0)$ position are shown in Tables 2 and 3.

\section{Analysis}

The data were analysed using the rotation diagram method. The molecular constants, the upper state energies and the partition functions required for applying this method were taken from the JPL line catalog (Pickett et al. 1998). This method gives the rotation temperature and total column density of a particular species if one knows the integrated line intensities of several lines with different upper state energies. The rotation temperatures and column densities estimated in this way are shown in Table 4. The rotation temperature is a lower limit to the gas kinetic temperature, and only constitutes a good measure of the kinetic temperature if the lines are thermalized. This is the case of the ammonia inversion lines which are thermalized with densities $n \sim 10^{3} \mathrm{~cm}^{-3}$. For this reason we used the $\mathrm{NH}_{3}$ inversion lines to estimate the gas kinetic temperature of the different envelope components.

For some molecules we have only observed one transition. In this case we calculated the total column density assuming optically thin emission, local thermodynamic equilibrium (LTE) and the rotation temperature derived from a molecule with similar excitation requirements. In these cases we have marked with the superindex "a" the rotation temperature in Table 4.

The molecules $\mathrm{NH}_{3}, \mathrm{~N}_{2} \mathrm{H}^{+}, \mathrm{CN}$ and $\mathrm{HCN}$ present hyperfine splitting. This allows us to derive the line opacity directly from the hyperfine line ratios. In these cases the column densities have been estimated directly from the line opacities. For $\mathrm{N}_{2} \mathrm{H}^{+}$ and $\mathrm{NH}_{3}$ we also calculated the source size assuming the rotation temperature derived from the $\mathrm{NH}_{3}$ inversion lines when the opacity of the main component is determined. We made Large Velocity Gradient (LVG) calculations for $\mathrm{SiO}, \mathrm{CS}$ and $\mathrm{C}^{34} \mathrm{~S}$. These calculations are shown in Tables 6 and 8. In all cases we have fitted the densities assuming a fixed kinetic temperature. The assumed gas kinetic temperatures are based on those derived from the $\mathrm{NH}_{3}$ data throughout Sect. 5.1 and are shown in Tables 6 and 8. We have used the CS collisional coefficients calculated by Green \& Chapman (1978) in the LVG calculations. The same collisional coefficients are used for SiO. This is a reasonable approximation since both molecules have the same mass and similar dipole moments. In the case of CS we were able to calculate the opacity and the source size because we had observed the main isotope and the rarer isotope $\mathrm{C}^{34} \mathrm{~S}$. All the column densities in Tables 4, 6 and 8 are beam-averaged column densities.

\section{Results}

\section{1. $\mathrm{NH}_{3}$}

We observed the ammonia $(1,1),(2,2),(3,3)$ and $(4,4)$ inversion transitions towards NGC 7129 - FIRS 2 and LkH $\alpha 234$. All the spectra are shown in Fig. 1. The $(1,1),(2,2)$ and $(3,3)$ lines were detected in both sources but only an upper limit has been obtained for the $(4,4)$ line. We fitted these lines using the $\mathrm{NH}_{3}$ procedure in the CLASS (this is the program dedicated to the analysis of single-dish spectra of the GILDAS software, http: //www . iram. fr/IRAMFR/GILDAS). This procedure fits all the hyperfine components assuming equal excitation temperature, central velocity and linewidth. The parameters given by the procedure are $\left(T_{\mathrm{ex}}-T_{\mathrm{bg}}\right) \times \tau_{\mathrm{m}}, V, \Delta V$, and $\tau_{\mathrm{m}}$ where $\tau_{\mathrm{m}}$ is the main group opacity and $T_{\mathrm{ex}}$ the excitation temperature (see Bachiller et al. 1987, for a more detailed description of this procedure). In case of optically thin emission $\tau_{\mathrm{m}}$ cannot be determined and is set arbitrarily to 0.1 . 
Table 4. LTE column densities.

\begin{tabular}{|c|c|c|c|c|c|c|c|c|c|}
\hline \multicolumn{5}{|c|}{ NGC 7129 - FIRS 2} & \multicolumn{5}{|c|}{$\mathrm{LkH} \alpha 234$} \\
\hline Molecule & Comp & $T_{\text {rot }}(\mathrm{K})$ & $N^{b}\left(\mathrm{~cm}^{-2}\right)$ & $\Omega_{s}$ & Molecule & Comp & $T_{\text {rot }}(\mathrm{K})$ & $N^{b}\left(\mathrm{~cm}^{-2}\right)$ & $\Omega_{s}$ \\
\hline \multirow[t]{2}{*}{$\mathrm{NH}_{3}$} & Cold & 13 & $4.9 \times 10^{14}$ & & $\mathrm{NH}_{3}$ & Cold & 22 & $4.0 \times 10^{13}$ & $8^{\prime \prime}$ \\
\hline & Warm & $31-87$ & $\sim 1.5 \times 10^{13}$ & & & Warm & $49-134$ & $\sim 1.1 \times 10^{13}$ & \\
\hline $\mathrm{N}_{2} \mathrm{H}^{+}$ & & $13^{a}$ & $3.8 \times 10^{13}$ & $\sim 21^{\prime \prime}$ & $\mathrm{N}_{2} \mathrm{H}^{+}$ & & $22^{a}$ & $1.0 \times 10^{13}$ & $\sim 8^{\prime \prime}$ \\
\hline $\mathrm{H}^{13} \mathrm{CO}^{+}$ & & $13^{a}$ & $2.2 \times 10^{12}$ & & $\mathrm{H}^{13} \mathrm{CO}^{+}$ & & $22^{a}$ & $1.7 \times 10^{12}$ & \\
\hline \multirow[t]{3}{*}{$\mathrm{CH}_{3} \mathrm{OH}$} & & 17 & $7.8 \times 10^{14}$ & & $\mathrm{CH}_{3} \mathrm{OH}^{p}$ & & 24 & $1.4 \times 10^{14}$ & \\
\hline & Hot core & $>80$ & $\sim 2.0 \times 10^{14}$ & & & & $>250$ & $\sim 4.0 \times 10^{14}$ & \\
\hline & & & & & $\mathrm{CH}_{3} \mathrm{OH}^{e}$ & & 59 & $4.8 \times 10^{14}$ & \\
\hline \multirow[t]{2}{*}{$\mathrm{H}_{2} \mathrm{CO}$} & Narrow & $\sim 10$ & $2.2 \times 10^{13}$ & & $\mathrm{H}_{2} \mathrm{CO}$ & & $\sim 11$ & $8.0 \times 10^{13}$ & \\
\hline & Wide & $\sim 9$ & $4.8 \times 10^{13}$ & & & & & & \\
\hline $\mathrm{H}_{2}{ }^{13} \mathrm{CO}$ & & $\sim 10^{a}$ & $2.3 \times 10^{12}$ & & $\mathrm{H}_{2}{ }^{13} \mathrm{CO}$ & & $\sim 11^{a}$ & $2.4 \times 10^{12}$ & \\
\hline $\mathrm{CH}_{3} \mathrm{CN}$ & Hot core & 63 & $3.6 \times 10^{12}$ & & $\mathrm{CH}_{3} \mathrm{CN}$ & & $<53$ & $>2.0 \times 10^{12}$ & \\
\hline $\mathrm{CN}$ & & 5 & $4.8 \times 10^{13}$ & & $\mathrm{CN}$ & & 6.5 & $6.1 \times 10^{13}$ & \\
\hline $\mathrm{HCN}$ & & $5^{a}$ & $1.6 \times 10^{13}$ & & $\mathrm{HCN}$ & & $6.5^{a}$ & $2.1 \times 10^{13}$ & \\
\hline $\mathrm{N}_{2} \mathrm{D}^{+}$ & & $13^{a}$ & $5.4 \times 10^{11}$ & & $\mathrm{~N}_{2} \mathrm{D}^{+}$ & & $22^{a}$ & $<2.3 \times 10^{11}$ & \\
\hline $\mathrm{DCO}^{+}$ & & 8.5 & $1.8 \times 10^{12}$ & & $\mathrm{DCO}^{+}$ & & 17 & $4.2 \times 10^{11}$ & \\
\hline $\mathrm{D}_{2} \mathrm{CO}$ & & $10^{a}$ & $2.0 \times 10^{12}$ & & $\mathrm{D}_{2} \mathrm{CO}$ & & $11^{a}$ & $3.7 \times 10^{12}$ & \\
\hline
\end{tabular}

${ }^{a}$ Assumed rotation temperature.

${ }^{p}$ Assuming a point source.

${ }^{e}$ Assuming a beam filling factor of 1 .

The fits to the $\mathrm{NH}_{3}$ lines in NGC 7129-FIRS 2 are shown in Table 2. The $\mathrm{NH}_{3}$ emission is optically thin in all the lines. However, the linewidth of the $(3,3)$ line is almost a factor of 3 larger than the linewidths of the $(1,1)$ and $(2,2)$ lines (see Table 2 and Fig. 2). In addition, the central velocities of the lines are slightly lower than those of the lower energy lines. This suggests that the $(3,3)$ line arises in a different region than the $(1,1)$ and $(2,2)$ lines. This interpretation is strengthened by the $\mathrm{NH}_{3}$ rotational diagram (see Fig. 3 ). The three lines detected in NGC 7129-FIRS 2 cannot be fitted by one single straight line, which implies the existence of at least two gas components with different rotation temperatures, a cold component traced by the $(1,1)$ and $(2,2)$ lines and a hot component only detected with the $(3,3)$ line. Using the $(1,1)$ and $(2$, 2) lines, we derive a rotation temperature $T_{12}=13 \mathrm{~K}$ and a column density $N\left(\mathrm{NH}_{3}\right)=4.9 \times 10^{14} \mathrm{~cm}^{-2}$ for the cold component. Because of the lack of radiative transitions between different K-ladders, the ammonia inversion lines are good thermometers of dense clouds. In fact, detailed radiative transfer calculations for $\mathrm{NH}_{3}$ show that $T_{\mathrm{k}}=T_{12}$ for $T_{\mathrm{k}}<20 \mathrm{~K}$ (Danby et al. 1988). This low value of the kinetic temperature in the cold envelope implies that depletion could be important in this young protostar.

Since we have not detected the $(4,4)$ line, we can only derive lower and upper limits for the rotation temperature of the hot component. The lower limit is given for the excitation temperature between the $(1,1)$ and $(3,3)$ lines, and the upper limit is given for the excitation temperature between the $(3,3)$ line and the upper limit to the $(4,4)$ line. We find that the hot component has a rotation temperature $T_{\text {rot }} \sim 30-90 \mathrm{~K}$, which implies a lower limit of $50 \mathrm{~K}$ to the kinetic temperature of this component. We have estimated $N\left(\mathrm{NH}_{3}\right) \sim 1.5 \times 10^{13} \mathrm{~cm}^{-3}$ for the hot component. This value has been derived from the integrated intensity of the $(3,3)$ line assuming $T_{\text {rot }}=90 \mathrm{~K}$ and LTE conditions and is, in fact, a lower limit to the actual column density of the hot gas.

The same procedure has been repeated for the ammonia lines towards $\mathrm{LkH} \alpha$ 234. Similarly to NGC 7129 - FIRS 2, the ammonia emission towards $\mathrm{LkH} \alpha 234$ cannot be fitted with one single rotation temperature (see Fig. 3). However, in this case the linewidth of the $(3,3)$ line is more similar to those of the $(1,1)$ and $(2,2)$ lines (see Table 3$)$. The linewidths seem to increase monotonically with the energy of the transition, but there is no jump between the linewidth of the $(3,3)$ line and those of the others as in the case of the protostar. We have fitted a two-component model to the rotational diagram in $\mathrm{LkH} \alpha 234$ and obtained rotation temperatures of $\sim 22 \mathrm{~K}$ and $\sim 49-134 \mathrm{~K}$ for cold and hot components respectively. This implies a kinetic temperature of $T_{\mathrm{k}} \sim 28 \mathrm{~K}$ for the cold envelope (Danby et al. 1988) and a lower limit of $T_{\mathrm{k}}>100 \mathrm{~K}$ for the hot component. Contrary to FIRS 2 , the $(1,1)$ line is moderately thick in this source. This allows us to estimate the size of the $\mathrm{NH}_{3}$ emission. Assuming that the excitation temperature of the $(1,1)$ line is equal to the rotation temperature of the cold component, we obtain a size of $\approx 8^{\prime \prime}$, which is lower than the size of the clump in the $1.3 \mathrm{~mm}$ continuum emission.

\section{2. $\mathrm{N}_{2} \mathrm{H}^{+}, \mathrm{H}^{13} \mathrm{CO}^{+}$}

Recent studies in pre-stellar cores reveal that the widely used tracers of dense gas, $\mathrm{C}^{18} \mathrm{O}$ and $\mathrm{CS}$, are not adequate to trace dense cold clumps. Both are strongly depleted in the core at densities of a few $10^{4} \mathrm{~cm}^{-3}$ and $T_{\mathrm{k}}<20 \mathrm{~K}$ (Tafalla et al. 2004). Only nitrogenated molecules seem to be unaffected by 

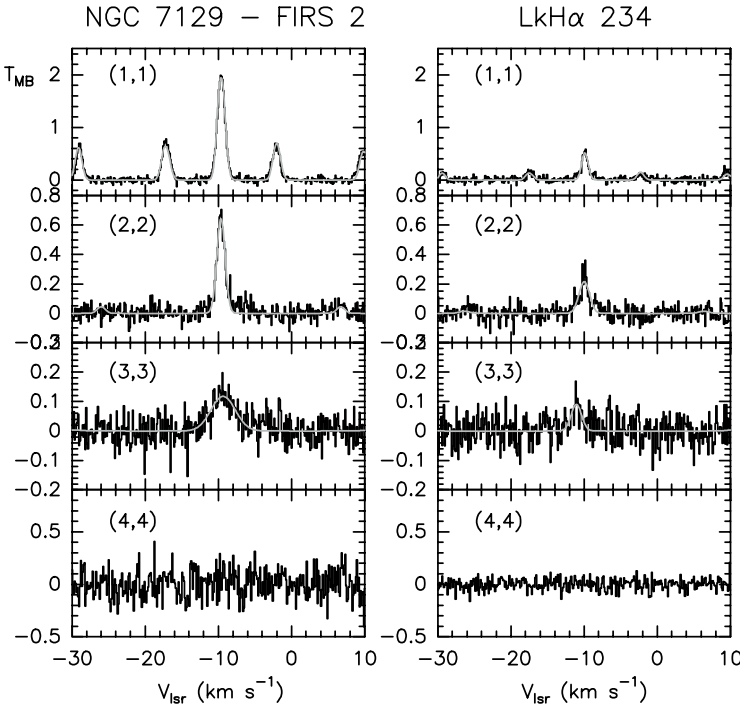

Fig. 2. Spectra of the $\mathrm{NH}_{3}(1,1),(2,2),(3,3)$ and $(4,4)$ inversion lines towards NGC 7129 - FIRS 2 (left) and LkH $\alpha 234$ (right).
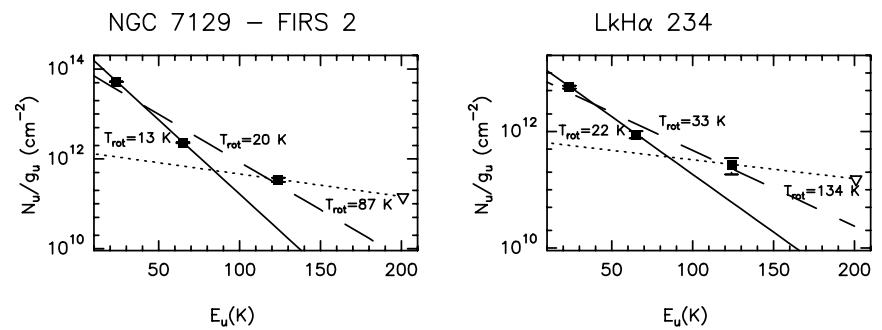

Fig. 3. Rotational diagram of $\mathrm{NH}_{3}$ in NGC 7129-FIRS 2 and $\mathrm{LkH} \alpha 234$. The long-dashed line corresponds to the fit assuming a single rotation temperature for all the transitions. The solid and dotted lines are the warm and cold component respectively of the twocomponent model. An empty inverted triangle indicates an upper limit.

depletion. In fact, $\mathrm{N}_{2} \mathrm{H}^{+}$seems to keep a constant abundance through the core and is an excellent tracer of the cold gas (e.g. Caselli et al. 1999; Tafalla et al. 2002).

We have made small maps in the $\mathrm{N}_{2} \mathrm{H}^{+} 1 \rightarrow 0$ and the $\mathrm{H}^{13} \mathrm{CO}^{+} 1 \rightarrow 0$ lines toward FIRS 2 and $\mathrm{LkH} \alpha 234$. In addition we have observed the $\mathrm{HC}^{18} \mathrm{O}^{+} 1 \rightarrow 0$ line towards the star position to have an estimate of the opacity of the $\mathrm{H}^{13} \mathrm{CO}^{+} 1 \rightarrow$ 0 line. In Figs. 4 and 5 we show the $\mathrm{N}_{2} \mathrm{H}^{+} 1 \rightarrow 0$ and the $\mathrm{H}^{13} \mathrm{CO}^{+} 1 \rightarrow 0$ line integrated intensity maps together with the continuum map at $1.3 \mathrm{~mm}$, and in Tables 2 and 3 we show the fits to the molecular lines. Because of the splitting of the $\mathrm{N}_{2} \mathrm{H}^{+} 1 \rightarrow 0$ line we can make an estimate of the line opacity using the same method as in the case of $\mathrm{NH}_{3}$.

Intense centrally-peaked emission is observed in the continuum map at $1.3 \mathrm{~mm}$ toward FIRS 2. The same morphology is observed in the $\mathrm{H}^{13} \mathrm{CO}^{+}$and $\mathrm{N}_{2} \mathrm{H}^{+}$maps, although the profile of the $\mathrm{H}^{13} \mathrm{CO}^{+}$emission is flatter than that of $\mathrm{N}_{2} \mathrm{H}^{+}$. To quantify this difference in the emission profile, we have calculated the line intensity ratio, $r_{1}=I_{\mathrm{m}}\left(\mathrm{N}_{2} \mathrm{H}^{+}\right) / I\left(\mathrm{H}^{13} \mathrm{CO}^{+}\right)$, where $I_{\mathrm{m}}\left(\mathrm{N}_{2} \mathrm{H}^{+}\right)$is the intensity of the main component of the $\mathrm{N}_{2} \mathrm{H}^{+} 1 \rightarrow 0$ line and $\mathrm{I}\left(\mathrm{H}^{13} \mathrm{CO}^{+}\right)$the intensity of the $\mathrm{H}^{13} \mathrm{CO}^{+}$ $1 \rightarrow 0$ line, in a radial strip at $0^{\prime \prime}$ offset in declination. The ratio $r_{1}$ changes from 2.7 at the center of the clump to 1.5 at an offset

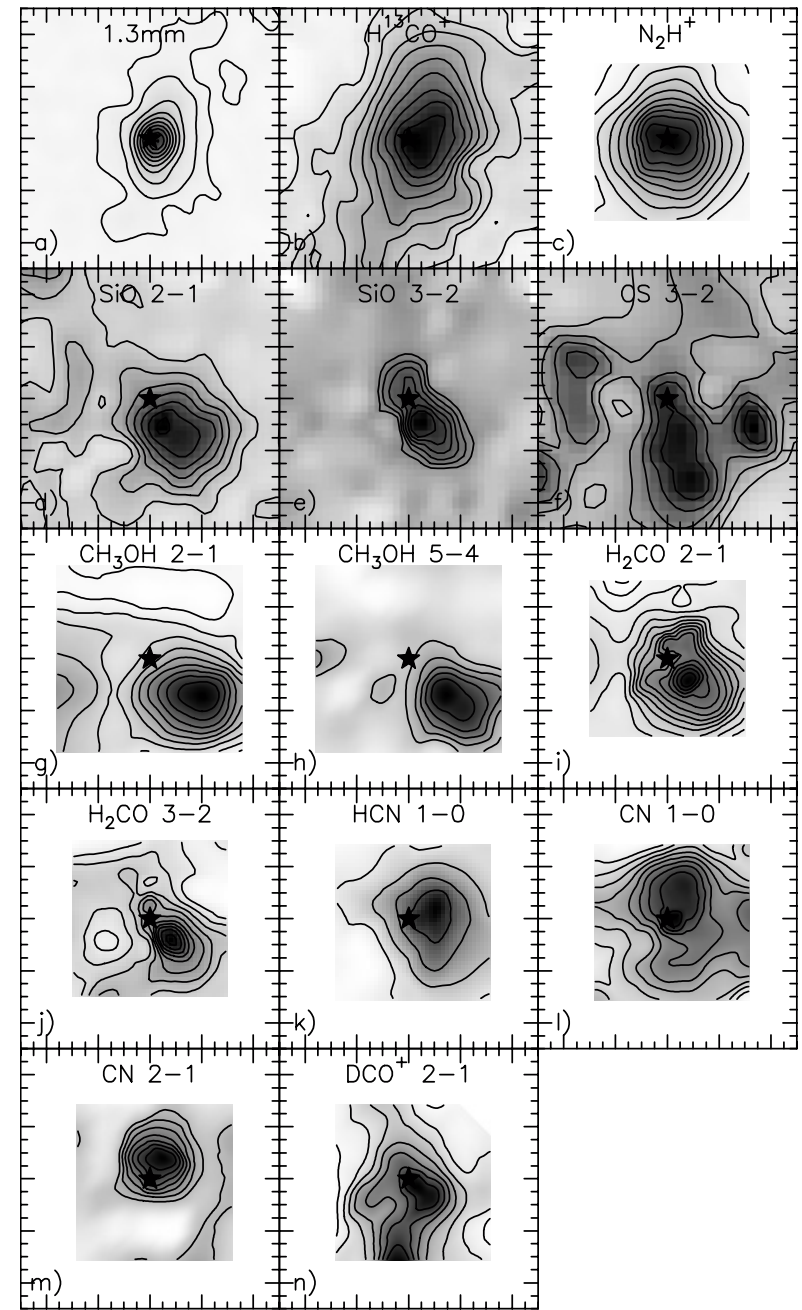

Fig. 4. Continuum map at $1.3 \mathrm{~mm}$ and integrated intensity maps of the observed lines in NGC 7129 - FIRS 2 . The box size is $100^{\prime \prime} \times 100^{\prime \prime}$ and is centered at the star position. Contour levels are: a) $39.2 \mathrm{mJy} / \mathrm{beam}$, and from 78.5 to $706.2 \mathrm{mJy} /$ beam by $78.5 \mathrm{mJy} /$ beam; b) 0.19 to 1.7 by $0.19 \mathrm{~K} \mathrm{~km} \mathrm{~s}^{-1}$; c) 0.2 to 8.2 by $0.8 \mathrm{~K} \mathrm{~km} \mathrm{~s}^{-1}$; d) 0.25 to 4 by $0.5 \mathrm{~K} \mathrm{~km} \mathrm{~s}^{-1}$; e) 1.5 to 4 by $0.5 \mathrm{~K} \mathrm{~km} \mathrm{~s}^{-1}$; f) 1.5 to 13.5 by $1.0 \mathrm{~K} \mathrm{~km} \mathrm{~s}^{-1}$; g) 2 to 20 by $2 \mathrm{~K} \mathrm{~km} \mathrm{~s}^{-1}$; h) 0.1 to 0.5 by $0.1 \mathrm{~K} \mathrm{~km} \mathrm{~s}^{-1}$; i) 1 to 14 by $1 \mathrm{~K} \mathrm{~km} \mathrm{~s}^{-1}$; j) 1 to 21 by $2 \mathrm{~K} \mathrm{~km} \mathrm{~s}^{-1}$; k) 2 to 7 by $1 \mathrm{~K} \mathrm{~km} \mathrm{~s}^{-1}$; l) 0.2 to 1.9 by $0.2 \mathrm{~K} \mathrm{~km} \mathrm{~s}^{-1}$; m) 0.8 to 3.4 by $0.4 \mathrm{~K} \mathrm{~km} \mathrm{~s}^{-1}$; n) 0.2 to 3 by $0.4 \mathrm{~K} \mathrm{~km} \mathrm{~s}^{-1}$.

of $\left(-30^{\prime \prime}, 0^{\prime \prime}\right)$. Using the LTE approximation with $T_{\text {rot }}=13 \mathrm{~K}$ (based on our $\mathrm{NH}_{3}$ calculations) and assuming optically thin emission in the $\mathrm{H}^{13} \mathrm{CO}^{+}$line, we derive that the $\mathrm{N}_{2} \mathrm{H}^{+} / \mathrm{H}^{13} \mathrm{CO}^{+}$ abundance ratio changes from $\sim 17$ in the $(0,0)$ position to $\sim 5$ at $\left(-30^{\prime \prime}, 0\right)$. Thus, the abundance of $\mathrm{H}^{13} \mathrm{CO}^{+}$relative to $\mathrm{N}_{2} \mathrm{H}^{+}$ seems to decrease by a factor of $\sim 3$ towards the clump center. However, this change in the estimated $\mathrm{H}^{13} \mathrm{CO}^{+}$abundance could be due to the larger opacity of the $\mathrm{H}^{13} \mathrm{CO}^{+}$line. To constrain the opacity of the $\mathrm{H}^{13} \mathrm{CO}^{+} 1 \rightarrow 0$ line, we observed the $\mathrm{HC}^{18} \mathrm{O}^{+} 1 \rightarrow 0$ line towards the $(0,0)$ position. The $\mathrm{H}^{13} \mathrm{CO}^{+}$ $1 \rightarrow 0 / \mathrm{HC}^{18} \mathrm{O}^{+} 1 \rightarrow 0$ line intensity ratio is $\sim 11$, showing that the $\mathrm{H}^{13} \mathrm{CO}^{+} 1 \rightarrow 0$ line is optically thin at this position.

In the above calculations we have assumed that the rotation temperature is uniform in the whole strip. This assumption could be unrealistic since the density and kinetic temperature 


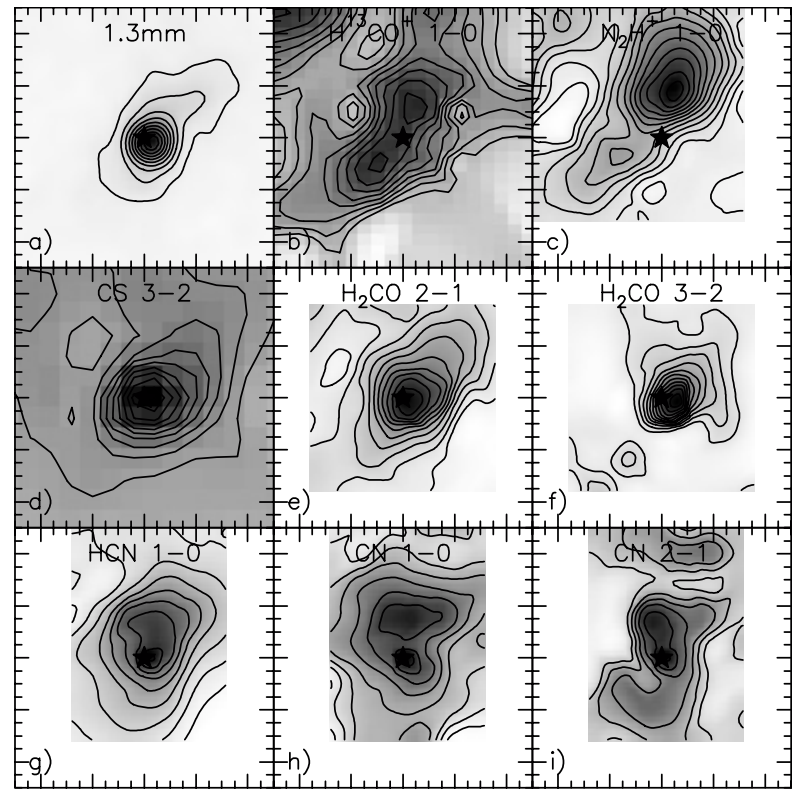

Fig. 5. Same as Fig. 1 for $\mathrm{LkH} \alpha$ 234. Contour levels are: a) $39.2 \mathrm{mJy} /$ beam, 78.5 to $706.2 \mathrm{mJy} /$ beam by steps of $78.5 \mathrm{mJy} / \mathrm{beam}$; b) 0.19 to 1.7 by $0.19 \mathrm{~K} \mathrm{~km} \mathrm{~s}^{-1}$; c) 0.2 to 8.2 by $0.4 \mathrm{~K} \mathrm{~km} \mathrm{~s}^{-1}$; d) 1.5 to 15 by $1.5 \mathrm{~K} \mathrm{~km} \mathrm{~s}^{-1}$; e) 1 to 11 by $1 \mathrm{~K} \mathrm{~km} \mathrm{~s}^{-1}$; f) 1.5 to 16.5 by $1.5 \mathrm{~K} \mathrm{~km} \mathrm{~s}^{-1}$; g) 2 to 11 by $1 \mathrm{~K} \mathrm{~km} \mathrm{~s}^{-1}$; h) 0.5 to 5 by $0.5 \mathrm{~K} \mathrm{~km} \mathrm{~s}^{-1}$; i) 1 to 6 by $1 \mathrm{~K} \mathrm{~km} \mathrm{~s}^{-1}$.

are expected to decrease with the distance from the star. We have used an LVG code to investigate if the different values of $r_{1}$ are due to a gradient in the physical conditions in the clump or/and the result of a change in the relative abundance of the two molecules. Since we are comparing the ground state lines of the two species, the ratio $r_{1}$ is very little dependent on the kinetic temperature and depends mainly on the hydrogen density and the $\mathrm{N}_{2} \mathrm{H}^{+} / \mathrm{H}^{13} \mathrm{CO}^{+}$abundance ratio. In Fig. 6 we show the ratio $r_{1}$ and the opacity of the main hyperfine $\mathrm{N}_{2} \mathrm{H}^{+}$ $1 \rightarrow 0$ line for a wide range of physical conditions assuming $X=\mathrm{N}_{2} \mathrm{H}^{+} / \mathrm{H}^{13} \mathrm{CO}^{+}=3,7,15$. The values of the opacity and $r_{1}$ measured towards the star position can only be fitted assuming $X=15$ (see Fig. 6). A lower value of $X$ would imply that the $\mathrm{N}_{2} \mathrm{H}^{+}$line is optically thin, in contradiction with our observational results. On the contrary, the value measured at the offset $\left(-30^{\prime \prime}, 0^{\prime \prime}\right)$ can only be fitted with $X<7$. Thus we conclude that the gradient in the value of $r_{1}$ cannot be due to the expected gradient in the excitation temperature of the observed lines across the clump, but must be due to a gradient in the $\mathrm{N}_{2} \mathrm{H}^{+} / \mathrm{H}^{13} \mathrm{CO}^{+}$abundance ratio.

As commented above, detailed studies in pre-stellar clumps show that the abundance of $\mathrm{N}_{2} \mathrm{H}^{+}$remains constant in these cold clumps while $\mathrm{H}^{13} \mathrm{CO}^{+}$could suffer from depletion in the densest part (Lee et al. 2003; Caselli et al. 2002). Assuming that $\mathrm{N}_{2} \mathrm{H}^{+}$has a constant abundance in the clump, we need to assume an $\mathrm{H}^{13} \mathrm{CO}^{+}$depletion factor $\gtrsim 2$ to fit our observations.

The integrated intensity maps of the $\mathrm{H}^{13} \mathrm{CO}^{+} 1 \rightarrow 0$ and $\mathrm{N}_{2} \mathrm{H}^{+} 1 \rightarrow 0$ lines towards LkH $\alpha 234$ are shown in Fig. 5. The $\mathrm{N}_{2} \mathrm{H}^{+} 1 \rightarrow 0$ map presents a very different morphology to that of the continuum and the $\mathrm{H}^{13} \mathrm{CO}^{+}$maps. While the continuum map presents a intense point-like emission at the position of
Table 5. Beam-averaged $\mathrm{N}_{2} \mathrm{H}^{+}$and $\mathrm{H}^{13} \mathrm{CO}^{+}$column densities.

\begin{tabular}{|c|c|c|c|c|}
\hline Source & $\begin{array}{l}T_{\text {rot }}(\mathrm{K}) \\
(\mathrm{K})\end{array}$ & $\begin{array}{c}N\left(\mathrm{H}^{13} \mathrm{CO}^{+}\right) \\
\left(\mathrm{cm}^{-2}\right)\end{array}$ & $\begin{array}{c}N\left(\mathrm{~N}_{2} \mathrm{H}^{+}\right) \\
\left(\mathrm{cm}^{-2}\right)\end{array}$ & $\frac{\mathrm{N}_{2} \mathrm{H}^{+}}{\mathrm{H}^{13} \mathrm{CO}^{+}}$ \\
\hline FIRS 2 & 13 & $2.2 \times 10^{12}$ & $3.8 \times 10^{13}$ & 17 \\
\hline$(-30,0)$ & 13 & $8.0 \times 10^{11}$ & $4.2 \times 10^{12}$ & 5 \\
\hline $\mathrm{LkH} \alpha 234$ & 22 & $1.7 \times 10^{12}$ & $1.0 \times 10^{13}$ & 6 \\
\hline$(-6,+18)$ & 10 & $8.8 \times 10^{11}$ & $8.5 \times 10^{12}$ & $\sim 10$ \\
\hline
\end{tabular}

$\mathrm{LkH} \alpha 234$, the $\mathrm{N}_{2} \mathrm{H}^{+}$map peaks at the offset $\left(-6^{\prime \prime},+18^{\prime \prime}\right)$. The map of the $\mathrm{H}^{13} \mathrm{CO}^{+}$line shows both peaks. As we will argue in the following, these different morphologies can only be explained if one assumes a different temperature and chemistry between both peaks.

In Table 4 we show the estimated $\mathrm{N}_{2} \mathrm{H}^{+}$and $\mathrm{H}^{13} \mathrm{CO}^{+}$column densities in LKH $\alpha 234$ assuming a rotation temperature of $22 \mathrm{~K}$ derived from the $\mathrm{NH}_{3}$ observations. We obtained $N\left(\mathrm{~N}_{2} \mathrm{H}^{+}\right) \sim 1.0 \times 10^{13} \mathrm{~cm}^{-2}$ and a $\mathrm{N}_{2} \mathrm{H}^{+} / \mathrm{H}^{13} \mathrm{CO}^{+}$abundance ratio of $\sim 6$. The $\mathrm{N}_{2} \mathrm{H}^{+} / \mathrm{H}^{13} \mathrm{CO}^{+}$ratio in $\mathrm{LkH} \alpha 234$ is lower than that found in FIRS 2.

We repeated the calculations for the offset $\left(-6^{\prime \prime},+18^{\prime \prime}\right)$. Continuum observations suggest that this clump is colder than that associated to $\mathrm{LkH} \alpha$ 234. In fact, if we assume a uniform $\mathrm{N}_{2} \mathrm{H}^{+}$abundance in the region and a typical dust temperature of $30 \mathrm{~K}$ towards $\mathrm{LkH} \alpha 234$, we need to assume a dust temperature $\$ 10 \mathrm{~K}$ at the offset $\left(-6^{\prime \prime},+18^{\prime \prime}\right)$ to explain the measured continuum flux. Thus, we have assumed a lower rotation temperature, $T_{\text {rot }} \sim 10 \mathrm{~K}$, in our LTE calculations. With these assumptions we obtain an $\mathrm{N}_{2} \mathrm{H}^{+}$column density of $\sim 1.3 \times 10^{13} \mathrm{~cm}^{-2}$ and an $\mathrm{N}_{2} \mathrm{H}^{+} / \mathrm{H}^{13} \mathrm{CO}^{+}$abundance ratio of $\sim 10$. This value is intermediate between those measured in LkH $\alpha 234$ and FIRS 2. However, the difference is a factor of 2 which is within the uncertainty involved in this kind of calculations. From our results, we can propose an evolutionary trend based on the $\mathrm{N}_{2} \mathrm{H}^{+} / \mathrm{H}^{13} \mathrm{CO}^{+}$ratio. This ratio is maximum in the IM Class 0 object FIRS 2 where molecular depletion is significant, it may take an intermediate value in the infrared low-mass star IRS 6 and is minimum in the envelope of the more evolved object, the $\mathrm{HBe}$ star LkH $\alpha 234$.

\subsection{CO outflows, SiO}

The $\mathrm{SiO}$ abundance is strongly enhanced (up to several orders of magnitude) in shocked regions. However, its abundance is very low in dark clouds and PDRs (Schilke et al. 2001). Because of this peculiarity, $\mathrm{SiO}$ is used as a diagnostic for shocks in both galactic and extragalactic regions (e.g. Bachiller et al. 1991; Martín-Pintado et al. 1992; García-Burillo et al. $2000,2001)$. In particular, it is commonly used to look for the energetic outflows associated with the youngest stellar objects. We have made maps of the $J=2 \rightarrow 1$ and $3 \rightarrow 2$ rotational lines of $\mathrm{SiO}$ around FIRS 2 and $\mathrm{LkH} \alpha 234$ to study the physical conditions and chemistry of the bipolar outflows found in these regions.

Interferometric and single-dish $\mathrm{CO} 2 \rightarrow 1$ observations show the existence of two bipolar outflows associated with FIRS 2. The axes of these outflows form an angle of almost 


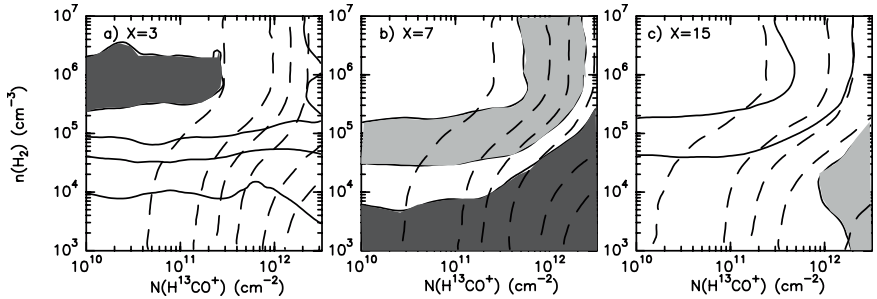

Fig. 6. Plots of the $r_{1}=I\left(\mathrm{~N}_{2} \mathrm{H}^{+}\right) / I\left(\mathrm{H}^{13} \mathrm{CO}^{+}\right)$ratio and opacity of the main $\mathrm{N}_{2} \mathrm{H}^{+}$hyperfine component (solid contours), and $\tau_{\mathrm{m}}$ (dashed contours), as a function of the $\mathrm{H}^{13} \mathrm{CO}^{+}$column density, $N\left(\mathrm{H}^{13} \mathrm{CO}^{+}\right)$, and hydrogen density, $n$, for a kinetic temperature of $T_{k}=15 \mathrm{~K}$ and an $\mathrm{N}_{2} \mathrm{H}^{+} / \mathrm{H}^{13} \mathrm{CO}^{+}$abundance ratio, $X=3,7,15$. We have shaded with dark grey the region with $r_{1}=1.5 \pm 0.3$ ( ratio measured at the $32^{\prime \prime}$ offset from the star position) and with a clear color $r_{1}=2.7 \pm 0.6$ (ratio measured at the star position). We need a depletion of at least a factor of 2 towards the center to fit both $\tau_{\mathrm{m}}$ and $r_{1}$ in both positions. Contour levels are: a) $r_{1}=0.8,1,1.2$ and $\tau_{\mathrm{m}}=0.1,0.5,1.0,2,5,10$; b) $r_{1}=1.8,2.2,2.7,3.2$ and $\tau_{\mathrm{m}}=0.1,0.5,1.0,2,5,10$; c) $r_{1}=3.2,5$, 7 and $\tau_{\mathrm{m}}=0.1,0.5,1.0,2,5,10$.

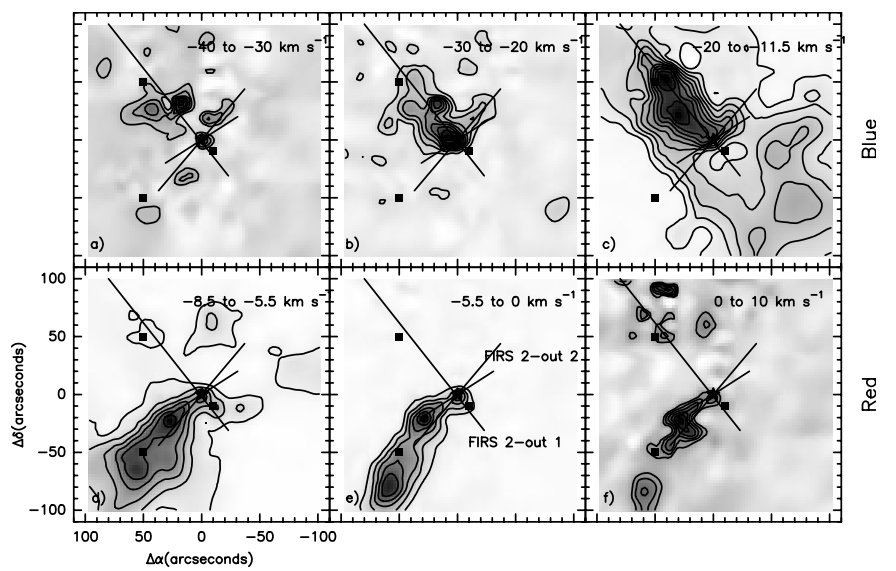

Fig. 7. High-velocity $\mathrm{CO}$ emission in FIRS 2. The blue-shifted velocities are shown in the top panels, and the red-shifted velocities in the bottom panels. Straight lines indicate the outflow axis. In the case of FIRS 2-out 2 we have drawn two axis because of outflow precession. Black squares show the positions shown in Table 3. Contour levels are: a) 1 to 6 by $1 \mathrm{~K} \mathrm{~km} \mathrm{~s}^{-1}$; b) 2 to 22 by $2 \mathrm{~K} \mathrm{~km} \mathrm{~s}^{-1}$; c) lev 7 to 70 by $7 \mathrm{~K} \mathrm{~km} \mathrm{~s}^{-1}$; d) 7 to 80 by $7 \mathrm{~K} \mathrm{~km} \mathrm{~s}^{-1}$; e) 4 to 50 by $4 \mathrm{~K} \mathrm{~km} \mathrm{~s}^{-1}$;f) 3 to 8 by $1 \mathrm{~K} \mathrm{~km} \mathrm{~s}^{-1}$.

$90^{\circ}$ giving a quadrupolar morphology to the spatial distribution of the high-velocity gas of the region. In Fig. 7 we show the maps of the high-velocity ${ }^{12} \mathrm{CO} 2 \rightarrow 1$ emission reported by Fuente et al. (2001). Interferometric observations show that the NE-SW outflow, hereafter FIRS 2-out 1, is the one associated with the millimeter source FIRS 2-MM1. An intense blue lobe is detected in the $\mathrm{CO} 2 \rightarrow 1$ emission in this outflow while the red lobe is only marginally detected. The NW-SE outflow, hereafter FIRS 2-out 2, seems to be associated with the infrared star, FIRS 2-IR (Fuente et al. 2001). In FIRS 2-out 2, the red lobe is more prominent than the blue one, giving a peculiar appearance to the high-velocity gas around FIRS 2 . The peaks of the ${ }^{12} \mathrm{CO}$ emission in this lobe present a "Z"-like shape which suggests that the axis of the outflow FIRS 2-out 2 is
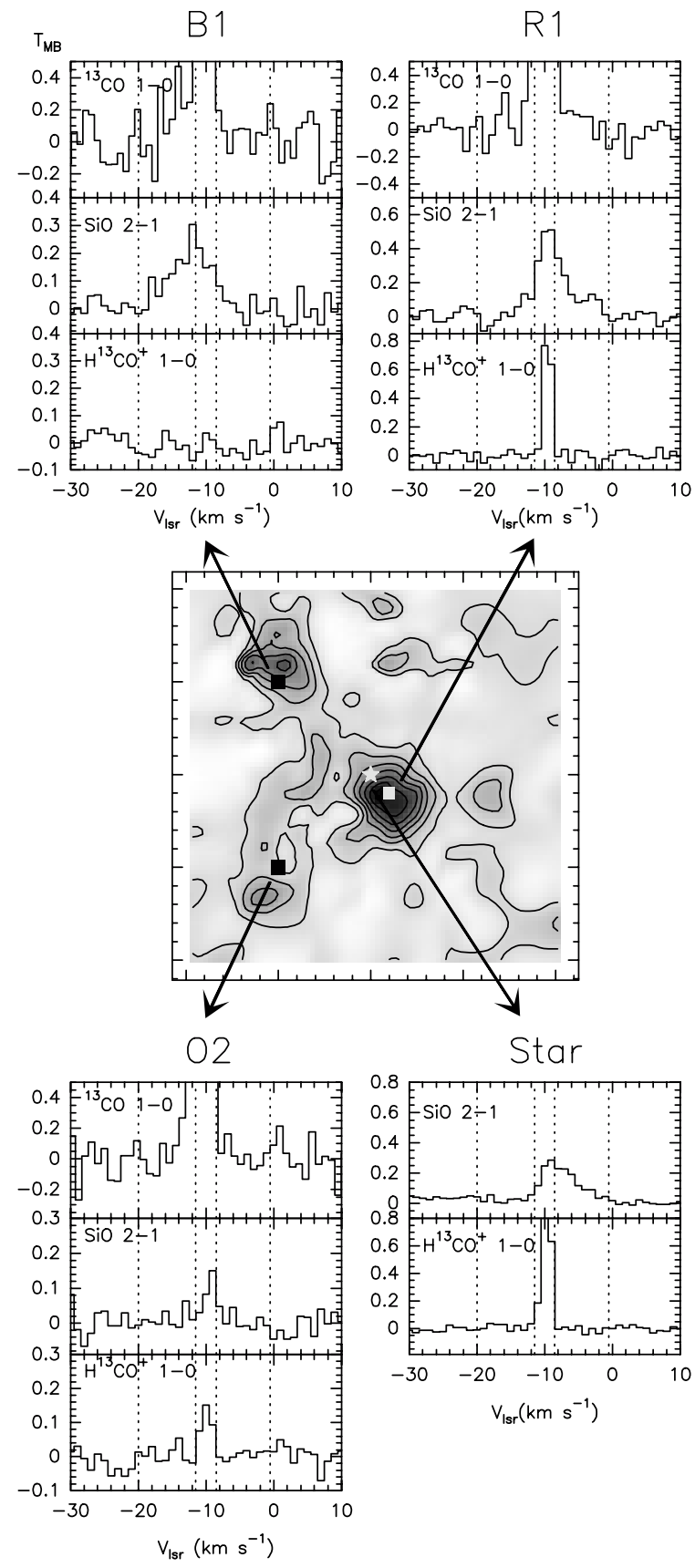

Fig. 8. In the central panel we show the integrated line intensity map of the $\mathrm{SiO} J=2 \rightarrow 1$ line toward FIRS 2. Contour levels are from $0.25 \mathrm{~K} \mathrm{~km} \mathrm{~s}^{-1}$ to $4 \mathrm{~K} \mathrm{~km} \mathrm{~s}^{-1}$ in steps of $0.5 \mathrm{~K} \mathrm{~km} \mathrm{~s}^{-1}$. Surrounding the central panel, we show the observed spectra of the ${ }^{13} \mathrm{CO} 1 \rightarrow 0$, $\mathrm{SiO} 2 \rightarrow 1$ and $\mathrm{H}^{13} \mathrm{CO}^{+} 1 \rightarrow 0$ lines towards selected positions of outflows FIRS 2-out 1 (top panels) and FIRS 2-out 2 (bottom panels).

precessing. Precession has been found in bipolar outflows associated to young low-mass stars (Gueth et al. 1996).

In Fig. 8 we present the integrated intensity map of the $\mathrm{SiO} 2 \rightarrow 1$ line towards FIRS 2 . The $\mathrm{SiO}$ emission is only observed along the axis of the two outflows. Furthermore the SiO peaks are not located at the star position but in the bow shocks located at the end of the outflow lobes. Thus, the SiO emission seems to be closely related to the bipolar outflows. The spectra of the $\mathrm{SiO}$ lines towards some selected positions are 
Table 6. LVG calculations: SiO.

\begin{tabular}{|c|c|c|c|c|c|c|c|c|}
\hline Source & $\begin{array}{l}\text { Vel. range } \\
\left(\mathrm{km} \mathrm{s}^{-1}\right)\end{array}$ & $\begin{array}{c}T_{k}(\mathrm{~K}) \\
(\mathrm{K})\end{array}$ & $\begin{array}{c}n \\
\left(\mathrm{~cm}^{-3}\right)\end{array}$ & $\begin{array}{l}N\left({ }^{13} \mathrm{CO}\right) \\
\left(\mathrm{cm}^{-2}\right)\end{array}$ & $\begin{array}{l}N(\mathrm{SiO}) \\
\left(\mathrm{cm}^{-2}\right)\end{array}$ & $\begin{array}{c}N\left(\mathrm{H}^{13} \mathrm{CO}^{+}\right) \\
\left(\mathrm{cm}^{-2}\right)\end{array}$ & $\mathrm{SiO} /{ }^{13} \mathrm{CO}$ & $\mathrm{SiO} / \mathrm{H}^{13} \mathrm{CO}^{+}$ \\
\hline FIRS 2 (Star) & $\begin{array}{l}-13.5--11.5 \\
-11.5--8.5 \\
-8.5--4.5 \\
-4.5--0.5 \\
\text { Total }\end{array}$ & $\begin{array}{l}50 \\
15 \\
50 \\
50\end{array}$ & $\begin{array}{l}5 \times 10^{5} \\
5 \times 10^{5} \\
4 \times 10^{5} \\
1 \times 10^{6}\end{array}$ & & $\begin{array}{l}1.5 \times 10^{11} \\
1.1 \times 10^{12} \\
1.2 \times 10^{12} \\
4.7 \times 10^{11} \\
2.8 \times 10^{12}\end{array}$ & $\begin{array}{l}1.0 \times 10^{11} \\
1.5 \times 10^{12}\end{array}$ & & $\begin{array}{l}1.5 \\
0.7\end{array}$ \\
\hline $\begin{array}{l}\left(-10^{\prime \prime},-10^{\prime \prime}\right) \mathrm{R} 1 \\
\text { (FIRS 2-out } 1)\end{array}$ & $\begin{array}{l}-13.5--11.5 \\
-11.5--8.5 \\
-8.5--4.5 \\
-4.5--0.5 \\
\text { Total }\end{array}$ & $\begin{array}{l}50 \\
15 \\
50 \\
50\end{array}$ & $\begin{array}{r}5 \times 10^{5 *} \\
4 \times 10^{5} \\
4 \times 10^{5} \\
3 \times 10^{4}\end{array}$ & $\begin{array}{r}3 \times 10^{15} \\
4 \times 10^{16} \\
3 \times 10^{15} \\
3 \times 10^{14} \\
4.6 \times 10^{16}\end{array}$ & $\begin{array}{r}4 \times 10^{11} \\
3 \times 10^{12} \\
1.5 \times 10^{12} \\
1.5 \times 10^{12} \\
6.4 \times 10^{12}\end{array}$ & $\begin{array}{l}1 \times 10^{12} \\
1 \times 10^{12}\end{array}$ & $\begin{array}{c}0.0001 \\
0.00007 \\
0.0005 \\
0.005 \\
0.0001\end{array}$ & 6 \\
\hline $\begin{array}{l}\left(50^{\prime \prime}, 50^{\prime \prime}\right) \mathrm{B} 1 \\
(\text { FIRS 2-out 1) }\end{array}$ & $\begin{array}{l}-17.5--12.5 \\
-12.5--8.5 \\
-8.5--4.5 \\
\text { Total }\end{array}$ & $\begin{array}{l}50 \\
50 \\
50\end{array}$ & $\begin{array}{l}2 \times 10^{5} \\
2 \times 10^{5} \\
2 \times 10^{5}\end{array}$ & $\begin{array}{l}1.5 \times 10^{15} \\
5.5 \times 10^{16} \\
2.0 \times 10^{15} \\
5.8 \times 10^{16}\end{array}$ & $\begin{array}{l}1.5 \times 10^{12} \\
1.5 \times 10^{12} \\
2.0 \times 10^{11} \\
3.2 \times 10^{12}\end{array}$ & $<2 \times 10^{12}$ & $\begin{array}{c}0.001 \\
0.00003 \\
0.0001 \\
0.00005\end{array}$ & $>1$ \\
\hline $\begin{array}{l}(50,-50) \text { O2 } \\
\text { (FIRS 2-out 2) }\end{array}$ & $-11.5--8.5$ & 15 & $8 \times 10^{4}$ & & $1.0 \times 10^{11}$ & $3 \times 10^{11}$ & & 3 \\
\hline $\begin{array}{l}\mathrm{LkH} \alpha 234 \\
\text { (IRS 6-out 1) }\end{array}$ & $-12.5--8.5$ & 50 & $5 \times 10^{5 *}$ & $1.3 \times 10^{17}$ & $2.0 \times 10^{11}$ & $1.0 \times 10^{12}$ & 0.000001 & 0.2 \\
\hline
\end{tabular}

* Assumed value for the hydrogen density.

shown in Fig. 8. The $\mathrm{SiO} 2 \rightarrow 1$ line towards the star position presents two velocity components, a narrow component with $\Delta V \sim 1.6 \mathrm{~km} \mathrm{~s}^{-1}$ which is centered at the velocity of the ambient cloud, $V_{\mathrm{lsr}} \sim-10.0 \mathrm{~km} \mathrm{~s}^{-1}$, and a wide component, $\Delta V \sim$ $7.0 \mathrm{~km} \mathrm{~s}^{-1}$ which is centered at $V_{\mathrm{lsr}} \sim-7.0 \pm 1.0 \mathrm{~km} \mathrm{~s}^{-1}$. The component at $-7.0 \mathrm{~km} \mathrm{~s}^{-1}$ corresponds to a well defined highvelocity clump which peaks at the position $\left(-10^{\prime \prime},-10^{\prime \prime}\right)$ (see Fig. 8). Hereafter we will refer to this clump as R1. There exists a counterpart blue clump at a velocity $V_{\mathrm{lsr}} \sim-12.5 \pm 0.5 \mathrm{~km} \mathrm{~s}^{-1}$ which peaks at the position $\left(50^{\prime \prime}, 50^{\prime \prime}\right)$ (hereafter B1). At this position, the profile of the $\mathrm{SiO} 2 \rightarrow 1$ line only presents a wide component with $\Delta V \sim 7 \mathrm{~km} \mathrm{~s}^{-1}$. The high-velocity clumps $\mathrm{R} 1$ and B1 have well defined velocities and positions like the "bullets" found in low-mass stars (see Bachiller 1996). The jet-like morphology of the $\mathrm{SiO}$ emission along the outflow axis as well as the existence of bullets argues in favor of the youth of this outflow.

The component at the velocity of the ambient cloud, $V_{\mathrm{lsr}} \sim$ $-10.0 \mathrm{~km} \mathrm{~s}^{-1}$, is also present in FIRS 2-out 2. The emission of this narrow component in FIRS 2-out 2 surrounds the red lobe as detected in ${ }^{12} \mathrm{CO}$. In fact, the narrow component is located adjacent to the peaks of the high-velocity $\mathrm{CO}$ emission, suggesting that $\mathrm{SiO}$ emission traces the molecular cloud gas entrained in the outflow. Narrow $\mathrm{SiO}$ components in the vicinity of the bipolar outflows have been detected in other Class 0 protostars (Codella et al. 1999). We propose an interpretation in which the morphology of the $\mathrm{SiO}$ emission is related to the evolutionary stage of the outflow. In FIRS 2-out 1 the SiO emission has a jet-like morphology and is concentrated in "bullets" ejected by the exciting star. This "jet-like" morphology is also observed in the interferometric ${ }^{12} \mathrm{CO}$ image reported by Fuente et al. (2001). The $\mathrm{SiO}$ emission in FIRS 2-out 2 surrounds the red $\mathrm{CO}$ lobe. We propose that in this case the $\mathrm{SiO}$ emission traces the material adjacent to the cavity walls excavated by the outflow which is being entrained into the outflow. The different profiles of the $\mathrm{SiO}$ emission in FIRS 2-out 1 and FIRS 2-out 2 are clearly seen in Fig. 8.

We calculated the $\mathrm{SiO} 3 \rightarrow 2 / 2 \rightarrow 1$ line intensity ratio, $r_{32}$, at the $\left(0^{\prime \prime}, 0^{\prime \prime}\right)$ position by degrading the angular resolution of the $\mathrm{SiO} 3 \rightarrow 2$ map to that of the $\mathrm{SiO} 2 \rightarrow 1$ one. A value of $r_{32} \sim 0.8$ is found in the three channels centered at the ambient velocity, while a value $r_{32}>1.0$ is found for the highvelocity gas. This reveals a higher excitation temperature for the high-velocity gas. In order to determine the physical conditions of both components we used an LVG code. Since $r_{32}$ suggests different physical conditions for the different velocity ranges, we carried out LVG calculations for the ambient, moderate velocity and high-velocity gas components (see Table 6). Taking into account the kinetic temperature derived from the $\mathrm{NH}_{3}$ lines we assumed $T_{k}=15 \mathrm{~K}$ for the ambient narrow component and $T_{k}=50 \mathrm{~K}$ for the high-velocity components. The derived densities and column densities are shown in Table 6. We are aware that the $\mathrm{NH}_{3}$ and $\mathrm{SiO}$ emissions could arise in different regions and that the assumed kinetic temperatures are very uncertain. But since the $\mathrm{SiO}$ lines are optically thin, the derived column densities are weakly dependent on the assumed kinetic temperature and are accurate within a factor of $\sim 2$. The values of Table 6 clearly show that the $\mathrm{SiO}$ abundance is larger by almost 2 orders of magnitude in the high-velocity gas of FIRS 2-out 1 than in the narrow component associated with 
FIRS 2-out 2. Within FIRS 2-out 1, we also detect a gradient in the $\mathrm{SiO}$ abundance, the $\mathrm{SiO} /{ }^{13} \mathrm{CO}$ ratio being 2 orders of magnitude larger in the high-velocity component than in the ambient component. This enhancement of the $\mathrm{SiO}$ abundance in the high-velocity gas is also observed in very young low-mass protostellar outflows (Bachiller et al. 1991, 2001) and interpreted as due to the chemical gas processing by the energetic shocks associated with the high-velocity "bullets".

Hydrogen densities are also quite independent of the assumed temperature for $T_{\mathrm{k}}>50 \mathrm{~K}$. The estimated hydrogen density decreases by only a factor of $\sim 4$ if we change the kinetic temperature from $15 \mathrm{~K}$ to $100 \mathrm{~K}$. Thus, the estimated hydrogen densities are accurate within a factor of $\sim 4$. Our calculations show that the density seems to increase from a few $10^{5} \mathrm{~cm}^{-3}$ in the ambient component to $>10^{6} \mathrm{~cm}^{-3}$ in the highvelocity gas at the star position. We have also carried out LVG calculations for R1 and B1, and at the offset $\left(50^{\prime \prime},-50^{\prime \prime}\right)$ where only the narrow component has been detected. The density in the bullets $\mathrm{R} 1$ and $\mathrm{B} 1$ is $\gtrsim 10^{5} \mathrm{~cm}^{-3}$ while the density in the narrow component detected at the offset $\left(50^{\prime \prime},-50^{\prime \prime}\right)$ is a few $10^{4} \mathrm{~cm}^{-3}$. This density is an upper limit to the hydrogen density in the narrow component, since the kinetic temperature is never expected to be lower than $15 \mathrm{~K}$. Thus, we consider that the estimated difference in the hydrogen density of the wide and narrow components is reliable. The lower density of the narrow component also supports our interpretation that the $\mathrm{SiO}$ emission arises in the gas of the molecular cloud surrounding the outflow.

We observed a map of $80^{\prime \prime} \times 80^{\prime \prime}$ around $\mathrm{LkH} \alpha 234$ in the $\mathrm{SiO} 2 \rightarrow 1$ and $3 \rightarrow 2$ lines. We detected $\mathrm{SiO}$ emission only towards the star position. Since we integrated for twice as much time towards the star position as in the other map positions, we cannot exclude the possibility of $\mathrm{SiO}$ emission at the same level in other positions. Thus we have poor information about the spatial distribution of the emission. Regarding the kinematical information, the large linewidth of the $\mathrm{SiO}$ lines, $\Delta V>3 \mathrm{~km} \mathrm{~s}^{-1}$, compared to that of the $(1,1)$ and $(2,2)$ ammonia lines suggests that the emission arises in the warm component. The weakness of the $\mathrm{SiO} 2 \rightarrow 1$ line emission as well as the lack of information about the source size makes any estimate of the hydrogen density very uncertain. We derived the $\mathrm{SiO}$ column density assuming $n \sim 5 \times 10^{5} \mathrm{~cm}^{-3}$. With this assumption we derive a $\mathrm{SiO}$ column density of $\sim 10^{11} \mathrm{~cm}^{-2}$. This value is lower by a factor of $>6$ than the total $\mathrm{SiO}$ column density towards FIRS 2 . The $\mathrm{SiO} /{ }^{13} \mathrm{CO}$ ratio towards this star is similar to that found in PDRs (Schilke et al. 2001).

In Table 7 we present a summary of the $\mathrm{SiO}$ observations. There is a clear evolutionary trend in the $\mathrm{SiO}$ behavior. The youngest outflow, FIRS 2-out 1, presents intense $\mathrm{SiO}$ emission at high-velocity with $\mathrm{SiO}$ abundances as high as $\sim 10^{-8}$. Towards the more evolved outflow FIRS 2-out 2 we detected only a weak $\mathrm{SiO}$ line at ambient velocity. The $\mathrm{SiO}$ abundance in this component is $\sim 10^{-10}$, i.e., two orders of magnitude lower than the $\mathrm{SiO}$ abundance in the high-velocity gas associated with FIRS 2-out 1 but still larger than the $\mathrm{SiO}$ abundance in PDRs and dark clouds. Towards LkH $\alpha 234$ we have detected $\mathrm{SiO}$ emission at the ambient velocities with a fractional abundance of $10^{-12}$. This abundance is similar to that measured
Table 7. SiO emission

\begin{tabular}{lccc}
\hline \hline Outflow & Age $(\mathrm{Myr})$ & Component & $X(\mathrm{SiO})^{3}$ \\
\hline FIRS 2-out 1 & $0.003^{1}$ & High velocity & $\sim 10^{-8}$ \\
FIRS 2-out 2 & $0.005^{1}$ & Ambient & $\sim 10^{-10}$ \\
LkH $\alpha$ 234 & $\sim 0.1^{2}$ & Ambient & $\sim 10^{-12}-10^{-11}$ \\
\hline
\end{tabular}

${ }^{1}$ Estimated age of the outflow assuming a velocity of $15 \mathrm{~km} \mathrm{~s}^{-1}$ and the lobe sizes measured from Fig. 1.

${ }^{2}$ Stellar age from Fuente et al. (1998).

${ }^{3}$ Fractional abundance estimates assuming $X\left({ }^{13} \mathrm{CO}\right)=2 \times 10^{-6}$ and $X\left(\mathrm{H}^{13} \mathrm{CO}^{+}\right)=10^{-10}$.

in PDRs and could be associated with the PDR produced by $\mathrm{LkH} \alpha 234$.

This evolutionary trend confirms that $\mathrm{SiO}$ is a good tracer of energetic shocks. The $\mathrm{SiO}$ abundance is highly enhanced when the shocks are strong enough to release the silicon from the grains into the gas phase (Martín-Pintado et al. 1992). In a MHD shock model the release of $\mathrm{Si}$ to the gas phase requires $V_{\text {shock }}>40 \mathrm{~km} \mathrm{~s}^{-1}$ (Flower et al. 1996). This is consistent with the trend of having larger $\mathrm{SiO}$ abundances in the higher velocity gas. Because of projection effects, the velocity we measure is a lower limit to $V_{\text {shock}}$. As the protostar evolves, the outflow fades and the amount of high-velocity molecular gas decreases. This produces a decrease in the $\mathrm{SiO}$ abundance. When the bipolar molecular outflow stops, the $\mathrm{SiO}$ abundance around the star decreases to the typical value in PDRs.

\section{4. $C S, C^{34} S$}

We have made a map of the CS $3 \rightarrow 2$ emission towards FIRS 2 and $\mathrm{LkH} \alpha 234$. In addition we have observed the $\mathrm{C}^{34} \mathrm{~S} 2 \rightarrow 1$, $3 \rightarrow 2$ and $5 \rightarrow 4$ lines towards the $(0,0)$ position. The spectra towards the $(0,0)$ position are shown in Fig. 9 and the Gaussian fits are shown in Tables 2 and 3 . The integrated intensity maps of the CS $J=3 \rightarrow 2$ line are shown in Fig. 4 .

Similarly to the case of $\mathrm{SiO}$, the profiles of the CS and $\mathrm{C}^{34} \mathrm{~S}$ lines towards FIRS 2 present high-velocity wings at redshifted velocities. However, the terminal velocities of these wings are lower than those of the $\mathrm{SiO}$ lines. The velocity range of the CS emission is between -14 and $-5 \mathrm{~km} \mathrm{~s}^{-1}$, i.e., it is only detected at the ambient velocities and in the moderate velocity component of the $\mathrm{SiO}$ emission. The spatial distribution of this component in the CS $3 \rightarrow 2$ line is different from that of the same component of the $\mathrm{SiO}$ emission, suggesting that the two molecules might be tracing different gas components even when we compare the same velocity range.

We have carried out LVG calculations to estimate the physical conditions of the gas emitting in CS. Different line ratios are found at the ambient velocities and in the moderate velocity range in the $\mathrm{C}^{34} \mathrm{~S}$ lines (see Fig. 9). Thus, we estimated the physical conditions in the two velocity intervals. We derived a density $\geq 3 \times 10^{6} \mathrm{~cm}^{-3}$ for the ambient component in FIRS 2 . This density is larger by a factor $\sim 6$ than the density estimated from $\mathrm{SiO}$ lines. This difference is not due to the assumed kinetic temperature, since even assuming a kinetic temperature 

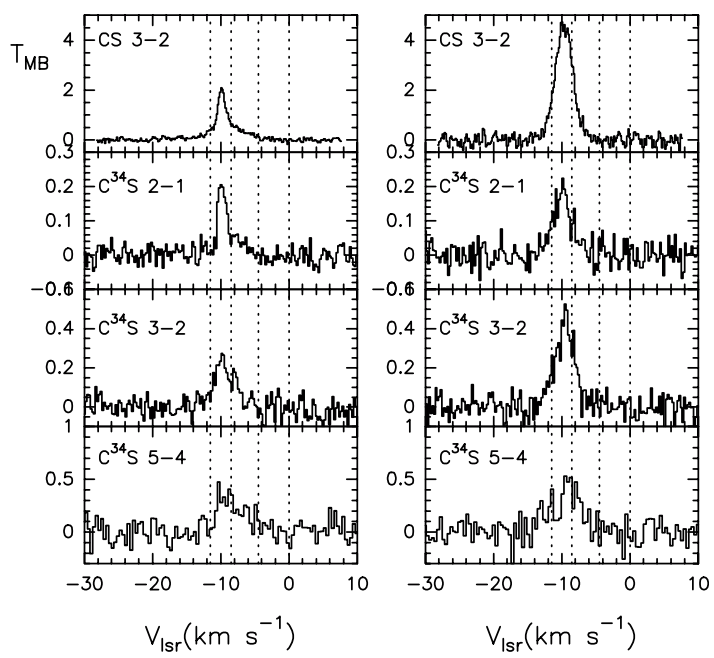

Fig. 9. Observed $\mathrm{CS}$ and $\mathrm{C}^{34} \mathrm{~S}$ spectra towards the $(0,0)$ position in NGC 7129 - FIRS 2 and $\mathrm{LkH} \alpha 234$.

of $100 \mathrm{~K}$ the density would decrease only by a factor of $\sim 4$. However, the densities derived from the $\mathrm{C}^{34} \mathrm{~S}$ lines in the moderate range are similar to those derived from the $\mathrm{SiO}$ lines. We propose that at least part of the $\mathrm{C}^{34} \mathrm{~S}$ line emission at ambient velocities does not arise in the outflow. We speculate on the possibility that the $\mathrm{C}^{34} \mathrm{~S}$ emission arises in a hot core.

The $\mathrm{CS}$ and $\mathrm{C}^{34} \mathrm{~S}$ lines observed in $\mathrm{LkH} \alpha 234$ are shown in Fig. 9. The linewidths of the $\mathrm{C}^{34} \mathrm{~S}$ lines are similar to those of the $\mathrm{SiO}$ and the $\mathrm{NH}_{3}(3,3)$ lines, suggesting that they trace a warm component. The emission of the CS lines is concentrated towards the position of the star, therefore the connection between the outflow and the CS emission is not clear. We derived a density $\sim 10^{5} \mathrm{~cm}^{-3}$ for this warm component. This density is lower than the typical density of the hot cores associated with massive stars. Since $\mathrm{LkH} \alpha 234$ is a visible star, the envelope has already been disrupted by the star and the UV radiation is escaping through the envelope. A dense $\left(n>10^{6} \mathrm{~cm}^{-3}\right)$ hot region similar to the hot cores in massive stars is not expected at this evolutionary stage.

\section{5. $\mathrm{CH}_{3} \mathrm{OH}$}

We observed up to 17 methanol lines towards the $(0,0)$ position in FIRS 2 and $\mathrm{LkH} \alpha$ 234. The large number of lines allows us to obtain a good estimate of the rotation temperature and the $\mathrm{CH}_{3} \mathrm{OH}$ column density. In addition, we have obtained a small map in the intense $\mathrm{CH}_{3} \mathrm{OH} 2 \rightarrow 1$ and $5 \rightarrow 4$ lines towards FIRS 2. The $\mathrm{CH}_{3} \mathrm{OH}$ maps around FIRS 2 are shown in Fig. 4. The $\mathrm{CH}_{3} \mathrm{OH}$ emission peaks towards the bullet $\mathrm{R} 1$, suggesting that these lines arise mainly in the molecular outflow.

The high-velocity resolution spectra of the $\mathrm{CH}_{3} \mathrm{OH}$ lines are shown in Fig. 10. The velocity profiles of the $\mathrm{CH}_{3} \mathrm{OH}$ lines towards FIRS 2 are similar to those found in the $\mathrm{SiO}$ lines. The low energy lines present two velocity components, a narrow component at the ambient velocity and a wide wing which extends to redshifted velocities and is centered at $-6 \pm$ $1 \mathrm{~km} \mathrm{~s}^{-1}$. In higher-energy transitions, the narrow component
NGC 7129 - FIRS 2

$\mathrm{LkH} \alpha 234$
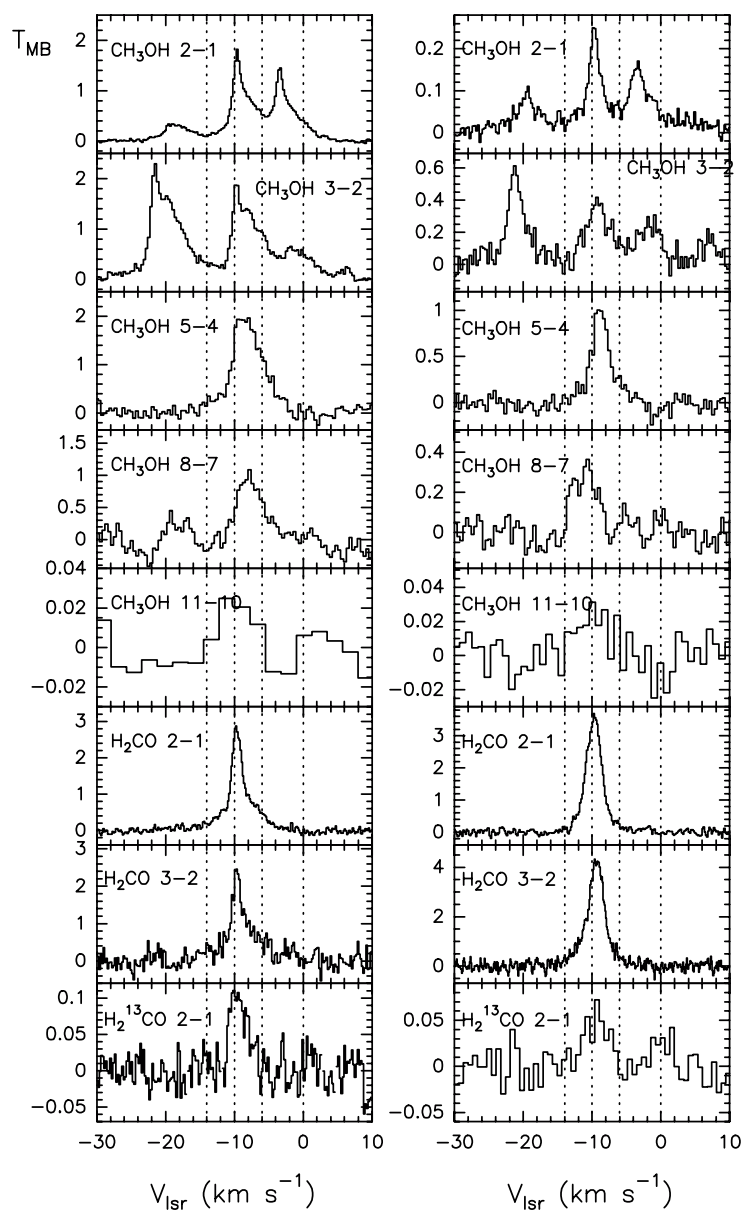

Fig. 10. Observed spectra of $\mathrm{CH}_{3} \mathrm{OH}$ and $\mathrm{H}_{2} \mathrm{CO}$ lines towards the $(0,0)$ position in NGC 7129-FIRS 2 and $\mathrm{LkH} \alpha 234$.

becomes weaker and only the wide redshifted one is detected. This is consistent with the integrated intensity maps of the $\mathrm{CH}_{3} \mathrm{OH} 2 \rightarrow 1$ and $5 \rightarrow 4$ lines shown in Fig. 4 which show a strong peak emission at the position of the bullet $\mathrm{R} 1$. In order to further investigate the nature of the $\mathrm{CH}_{3} \mathrm{OH}$ emission, we have fitted the lines with Gaussian profiles and made some correlations. In Fig. 11 we plot the central velocity and linewidths of the $\mathrm{CH}_{3} \mathrm{OH}$ lines vs. the upper state energy of the observed transition. It is clearly seen that the linewidths of the $\mathrm{CH}_{3} \mathrm{OH}$ lines increase with the upper state energy of the transition at moderate energies. In fact, they increase from $\sim 3 \mathrm{~km} \mathrm{~s}^{-1}$ in the low energy transitions to $\sim 7 \mathrm{~km} \mathrm{~s}^{-1}$ in transitions with $E_{u}>50 \mathrm{~K}$. But this trend is not present for higher energy lines, which seem to have a constant linewidth of $\sim 4 \mathrm{~km} \mathrm{~s}^{-1}$. A similar behavior is found when one compares the velocity of the line with the upper state energy. The line velocity changes from $V \sim-10 \mathrm{~km} \mathrm{~s}^{-1}$ to $\sim-7.5 \mathrm{~km} \mathrm{~s}^{-1}$ when the energy increases from $\sim 10 \mathrm{~K}$ to $50 \mathrm{~K}$. However, for higher energies, the line velocities seem to go in the opposite way and change from $\sim-8 \mathrm{~km} \mathrm{~s}^{-1}$ to $-10 \mathrm{~km} \mathrm{~s}^{-1}$. This suggests that the emission of the low and moderate energy transitions $\left(E_{u}>50 \mathrm{~K}\right)$ arises in the molecular outflow. The correlation found between the linewidth and the energy of the transition suggests that the 
Table 8. LVG calculations: $\mathrm{CS}, \mathrm{C}^{34} \mathrm{~S}$.

\begin{tabular}{clcccc}
\hline \hline Source & \multicolumn{1}{c}{ Vel. range } & $T_{\mathrm{k}}(\mathrm{K})$ & $n\left(\mathrm{~cm}^{-3}\right)$ & $N\left(\mathrm{C}^{34} \mathrm{~S}\right)\left(\mathrm{cm}^{-2}\right)$ & $\Omega_{s}$ \\
\hline NGC 7129 - FIRS 2 & $-10.5--9.5 \mathrm{~km} \mathrm{~s}^{-1}$ & 15 & $3 \times 10^{6}$ & $8.0 \times 10^{11}$ & $\sim 9^{\prime \prime}$ \\
& $-9.5--6.5 \mathrm{~km} \mathrm{~s}^{-1}$ & 50 & $6 \times 10^{5}$ & $7.1 \times 10^{11}$ & $\sim 4^{\prime \prime}$ \\
& Total & & & $1.5 \times 10^{12}$ & \\
LkH $\alpha$ 234 & $-11.5--9.5 \mathrm{~km} \mathrm{~s}^{-1}$ & 50 & $2 \times 10^{5}$ & $9.6 \times 10^{11}$ & $14^{\prime \prime}$ \\
& $-9.5--5.5 \mathrm{~km} \mathrm{~s}^{-1}$ & 50 & $4 \times 10^{5}$ & $7.8 \times 10^{11}$ & $6^{\prime \prime}$ \\
& Total & & & $1.7 \times 10^{12}$ & \\
\hline
\end{tabular}

NGC 7129 - FIRS 2
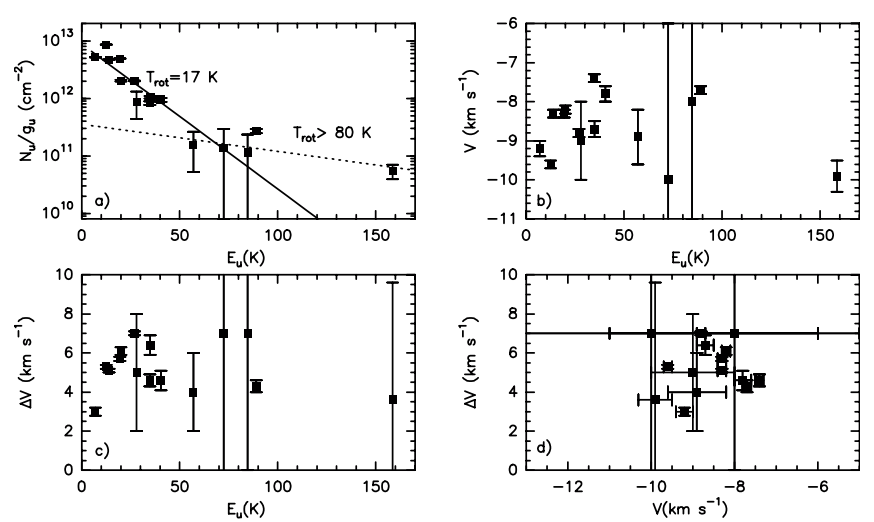

Fig. 11. The a) panel shows the rotational diagram for $\mathrm{CH}_{3} \mathrm{OH}$ in the $(0,0)$ position of NGC 7129 - FIRS 2 . We degraded the angular resolution of the $1.3 \mathrm{~mm}$ maps (the $5 \rightarrow 4$ and $8 \rightarrow 7$ methanol lines) to the angular resolution of the $3 \mathrm{~mm}$ maps (the $2 \rightarrow 1$ and $11 \rightarrow 10$ lines). Two rotation temperatures $\left(T_{\text {rot }}=17 \mathrm{~K}\right.$ and $T_{\text {rot }}>80 \mathrm{~K}$ are required to fit the data). In the b) and c) panels we plot the line velocities and linewidths, $V$ and $\Delta V$, of the $\mathrm{CH}_{3} \mathrm{OH}$ lines as given by the Gaussian fits shown in Table 3 versus the upper state energy of the observed transition $\left(E_{u}\right)$. Finally, the d) panel shows $V$ versus $\Delta V$ for the $\mathrm{CH}_{3} \mathrm{OH}$ lines. This panel clearly shows the existence of two velocity components, one at $\sim-10 \mathrm{~km} \mathrm{~s}^{-1}$ and the other at $\sim-8 \mathrm{~km} \mathrm{~s}^{-1}$ in this position.

high-velocity gas is associated with higher excitation temperatures. This result is consistent with the density estimated from the $\mathrm{SiO}$ lines. For $E_{u}>50 \mathrm{~K}$, there is a jump in the line velocity which returns to $\sim-10 \mathrm{~km} \mathrm{~s}^{-1}$. We propose that this could be due to the existence of a hot component in the $\mathrm{CH}_{3} \mathrm{OH}$ emission in addition to that related to the bipolar outflow.

In Fig. 11 we show the $\mathrm{CH}_{3} \mathrm{OH}$ rotational diagram for FIRS 2. The observed $\mathrm{CH}_{3} \mathrm{OH}$ transitions cannot all be fitted with a single straight line. We need to assume at least two rotation temperatures to fit the observational data. The low energy transitions $\left(E_{u}<50 \mathrm{~K}\right)$ are well fitted with a $T_{\text {rot }} \sim 17 \mathrm{~K}$, while the high energy transitions require a higher rotation temperature, $T_{\text {rot }}>80 \mathrm{~K}$. We propose the existence of a "hot core" component which dominates the emission in the high energy transitions.

Several $\mathrm{CH}_{3} \mathrm{OH}$ lines were also been observed in $\mathrm{LkH} \alpha 234$. In this case all the lines are centered at the ambient velocity. However, there are important variations in the linewidths of the observed lines. Like in the case of FIRS 2, the linewidths seem to increase with the energy of the upper level for $E_{u}<50 \mathrm{~K}$ (see Fig. 12). We have only detected the

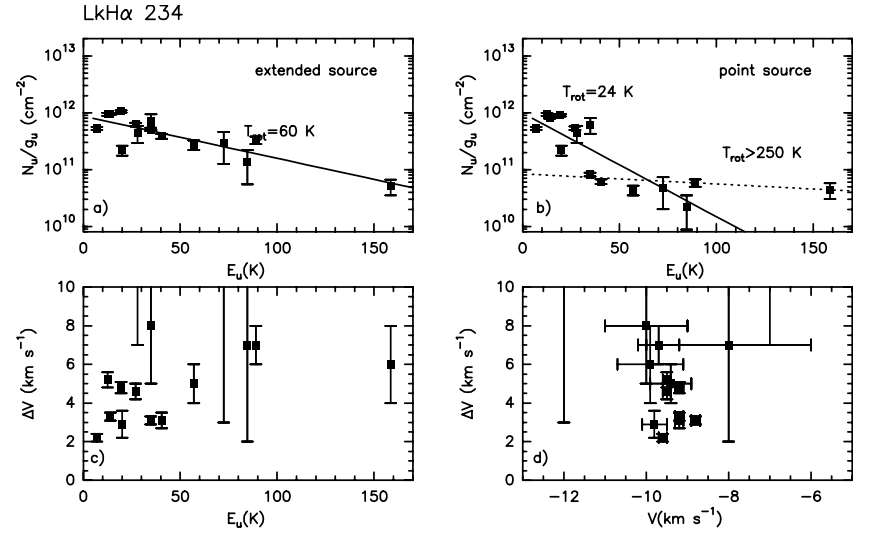

Fig. 12. The top panels show the rotational diagram for $\mathrm{CH}_{3} \mathrm{OH}$ in $\mathrm{LkH} \alpha 234$ in the two limit cases assumed in the paper; panel a) corresponds to a beam filling factor $\sim 1$ and panel b) corresponds to a point source. In the bottom panels we plot the linewidths, $\Delta V$, derived from the Gaussian fits versus the upper energy state of the transition, $E_{u}$, and the central velocity, $V$, for all the lines. In the case of $\mathrm{LkH} \alpha 234$ we have only one velocity component.

methanol lines towards the $(0,0)$ position and, consequently, we have no information about the size of the emitting region. Thus, we have considered the two limiting cases of a point source and a beam filling factor of 1 to make the rotational diagram. In the first case we need two gas components to fit all the observed transitions. The cold one would have $T_{\text {rot }} \sim 24 \mathrm{~K}$, and the hot one, $T_{\text {rot }}>250 \mathrm{~K}$. But if we assume a beam filling factor of 1 , all the observed transitions are well fit with $T_{\text {rot }} \sim 60 \mathrm{~K}$. With our data, we cannot distinguish between these two cases.

\section{6. $\mathrm{H}_{2} \mathrm{CO}$}

We observed two $\mathrm{H}_{2} \mathrm{CO}$ rotational transitions and one of the rarer isotope $\mathrm{H}_{2}{ }^{13} \mathrm{CO}^{+}$toward FIRS 2 and $\mathrm{LkH} \alpha$ 234. The obtained spectra are shown in Fig. 10. Similarly to other molecules, the $\mathrm{H}_{2} \mathrm{CO}$ spectra towards FIRS 2 present two well differentiated components, a narrow one centered at $\sim-9.6 \mathrm{~km} \mathrm{~s}^{-1}$ with a linewidth of $\sim 1.3 \mathrm{kms} \mathrm{s}^{-1}$ and a much wider one centered at $\sim-9.0 \mathrm{~km} \mathrm{~s}^{-1}$. However, the wide component does not have the typical R1 profile observed in the $\mathrm{SiO}, \mathrm{CS}$ and methanol lines at the star position. While the profiles of the $\mathrm{SiO}, \mathrm{CS}$ and methanol lines present only red wings, the $\mathrm{H}_{2} \mathrm{CO}$ lines present a quite symmetric profile with blue and red wings. Consequently, the central velocity of the wide $\mathrm{H}_{2} \mathrm{CO}$ component is similar to that of the ambient gas 


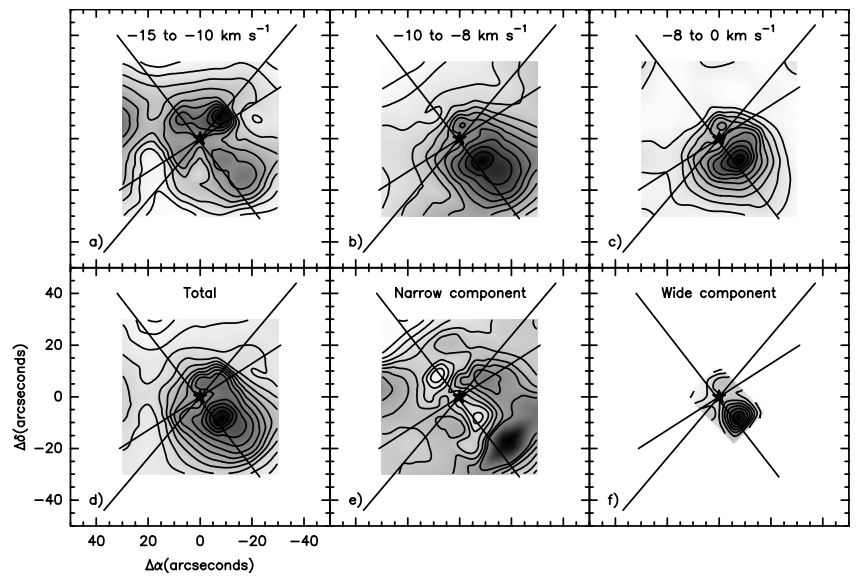

Fig. 13. Panels a)-d) are the integrated intensity maps of the $\mathrm{H}_{2} \mathrm{CO}$ $2_{12} \rightarrow 1_{11}$ line towards NGC 7129-FIRS 2 for different velocity intervals. In panel e) we show the integrated intensity map of the $\mathrm{H}_{2} \mathrm{CO}$ lines after subtracting the Gaussian fit to the wide component from all the spectra. In panel f) we show the area of this wide component as derived from the Gaussian fit. The axes of the outflow FIRS 2-out 1 and FIRS 2-out 2 are indicated. Contour levels are: a) 0.5 to 5 by $0.5 \mathrm{~K} \mathrm{~km} \mathrm{~s}^{-1}$; b) 1.0 to 6.5 by $0.5 \mathrm{~K} \mathrm{~km} \mathrm{~s}^{-1}$; c) 0.5 to 5 by $0.5 \mathrm{~K} \mathrm{~km} \mathrm{~s}^{-1}$; d) 1 to 14 by $1 \mathrm{~K} \mathrm{~km} \mathrm{~s}^{-1}$; e) 0.5 to 5 by $0.5 \mathrm{~K} \mathrm{~km} \mathrm{~s}^{-1}$; f) 1 to 11.5 by $1 \mathrm{~K} \mathrm{~km} \mathrm{~s}^{-1}$.

and the linewidth is as large as $\Delta V=7 \mathrm{~km} \mathrm{~s}^{-1}$. To further investigate the nature of these components we have studied the integrated intensity maps of the $\mathrm{H}_{2} \mathrm{CO} 2_{12} \rightarrow 1_{11}$ line for the different velocity intervals (see Fig. 13). The most striking feature could be the jet-like morphology observed in the $\mathrm{H}_{2} \mathrm{CO}$ emission at blue velocities (from -15 to $-10 \mathrm{~km} \mathrm{~s}^{-1}$ ). At red velocities, the emission is maximum at the offset $\left(-7^{\prime \prime},-7^{\prime \prime}\right)$ which is located close to the bullet R1. Since the wide component has a very well differentiated profile, we were been able to subtract the wide component from the observed spectra, and mapped both components separately. Our results are quite suggestive. The wide component presents a jet-like morphology with the maximum towards the position R1. The morphology of the narrow component is an intense ridge which surrounds the jet. This strongly suggests that the narrow component traces the shocked gas of the molecular cloud which is interacting with the jet. The maximum of this narrow component coincides with the position where the bullet R1 impinges on the cloud. We derived rotation temperatures and column densities in the narrow and wide components separately, and obtained similar excitation conditions in both components. Thus, although the kinematics is clearly different, the physical conditions of both components are quite similar.

In Fig. 10 we show the $\mathrm{H}_{2} \mathrm{CO}$ spectra towards $\mathrm{LkH} \alpha 234$. The profiles of the $\mathrm{H}_{2} \mathrm{CO}$ lines in this source also suggest the existence of a narrow and a wide component. However, these two components cannot be easily separated. For this reason we have derived rotation temperatures by considering the sum of the two components. We obtain a rotation temperature and $\mathrm{H}_{2} \mathrm{CO}$ column density similar to those obtained in FIRS 2. In Fig. 5 we show the integrated line intensity maps for this source. Similarly to the case of FIRS 2 we find emission along the outflow and in a direction perpendicular to it. Thus far, no
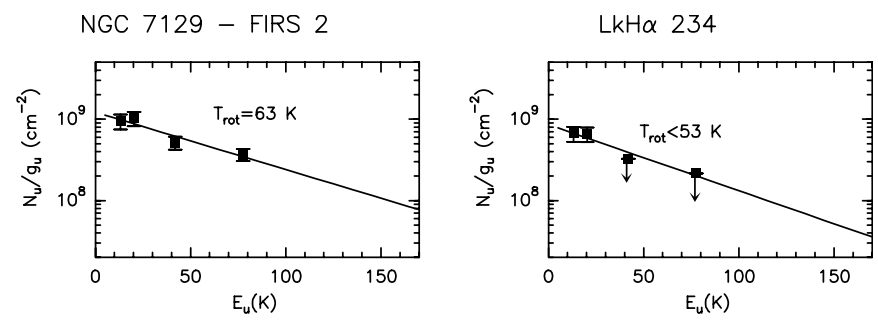

Fig. 14. Rotational diagram of $\mathrm{CH}_{3} \mathrm{CN}$ in the $(0,0)$ position of FIRS 2 and $\mathrm{LkH} \alpha 234$.

bipolar outflow has been detected in this direction. Thus, this $\mathrm{H}_{2} \mathrm{CO}$ emission is associated with the flattened clump in which the Herbig Be star LkH $\alpha 234$ is embedded, and which is being heated by the recently born star.

\section{7. $\mathrm{CH}_{3} \mathrm{CN}$}

We have observed the $\mathrm{CH}_{3} \mathrm{CN} 5 \rightarrow 4$ and $13 \rightarrow 12$ lines towards FIRS 2 and $\mathrm{LkH} \alpha$ 234. Because of the rotational structure of $\mathrm{CH}_{3} \mathrm{CN}$, one can observe several lines at different energies very close in frequency. This allows us to estimate the rotation temperature avoiding observational errors and the uncertainty due to the unknown source size. We have carried out these calculations towards our two sources. Unlike for the other molecules observed, we do not detect a cold component in the $\mathrm{CH}_{3} \mathrm{CN}$ lines, but only the warm one. The detection of a hot $\mathrm{CH}_{3} \mathrm{CN}$ component with $T_{\mathrm{k}}>63 \mathrm{~K}$ in FIRS 2 shows the existence of a hot core in this object.

$\mathrm{CH}_{3} \mathrm{CN}$ seems to be the best tracer of hot cores in these intermediate-mass stars. Contrary to $\mathrm{CH}_{3} \mathrm{OH}$ and $\mathrm{H}_{2} \mathrm{CO}$ whose low energy lines arise mainly in the bipolar outflow, the rotational lines of $\mathrm{CH}_{3} \mathrm{CN}$ seem to arise in the hot core and provide a good measure of the kinetic temperature of this hot component.

\section{8. $\mathrm{CN}, \mathrm{HCN}$}

We mapped a small region around FIRS 2 and $\mathrm{LkH} \alpha 234$ in the $\mathrm{HCN} 1 \rightarrow 0$ and $\mathrm{CN} 1 \rightarrow 0$ and $2 \rightarrow 1$ lines. The integrated intensity maps around FIRS 2 are shown in Figs. 4 and 5. Thus far, we have two different kinds of molecule depending on the morphology of their emission in FIRS 2. The first group is formed by the molecular ions $\mathrm{N}_{2} \mathrm{H}^{+}$and $\mathrm{H}^{13} \mathrm{CO}^{+}$, which peak at the YSO position and trace the bulk of the cold envelope. The second group is formed by $\mathrm{CS}, \mathrm{SiO}, \mathrm{CH}_{3} \mathrm{OH}$ and $\mathrm{H}_{2} \mathrm{CO}$ peaks at the position of $\mathrm{R} 1$, and its emission comprises an important contribution from the molecular outflow FIRS 2out 1 . The morphology of the $\mathrm{CN} 1 \rightarrow 0$ emission is different from that of the two groups mentioned. The $\mathrm{CN}$ emission does not peak at the star position nor at the position of the bullet R1, but to the north, suggesting a new chemical differentiation in the protostellar envelope. The morphology of the HCN emission is also different from that of the other molecular lines. In fact, the $\mathrm{HCN}$ emission peaks to the NW of the star position, in a position intermediate between the bullet $\mathrm{R} 1$ and the peak of the $\mathrm{CN}$ emission. The large linewidths of the $\mathrm{HCN} 1 \rightarrow 0$ line 

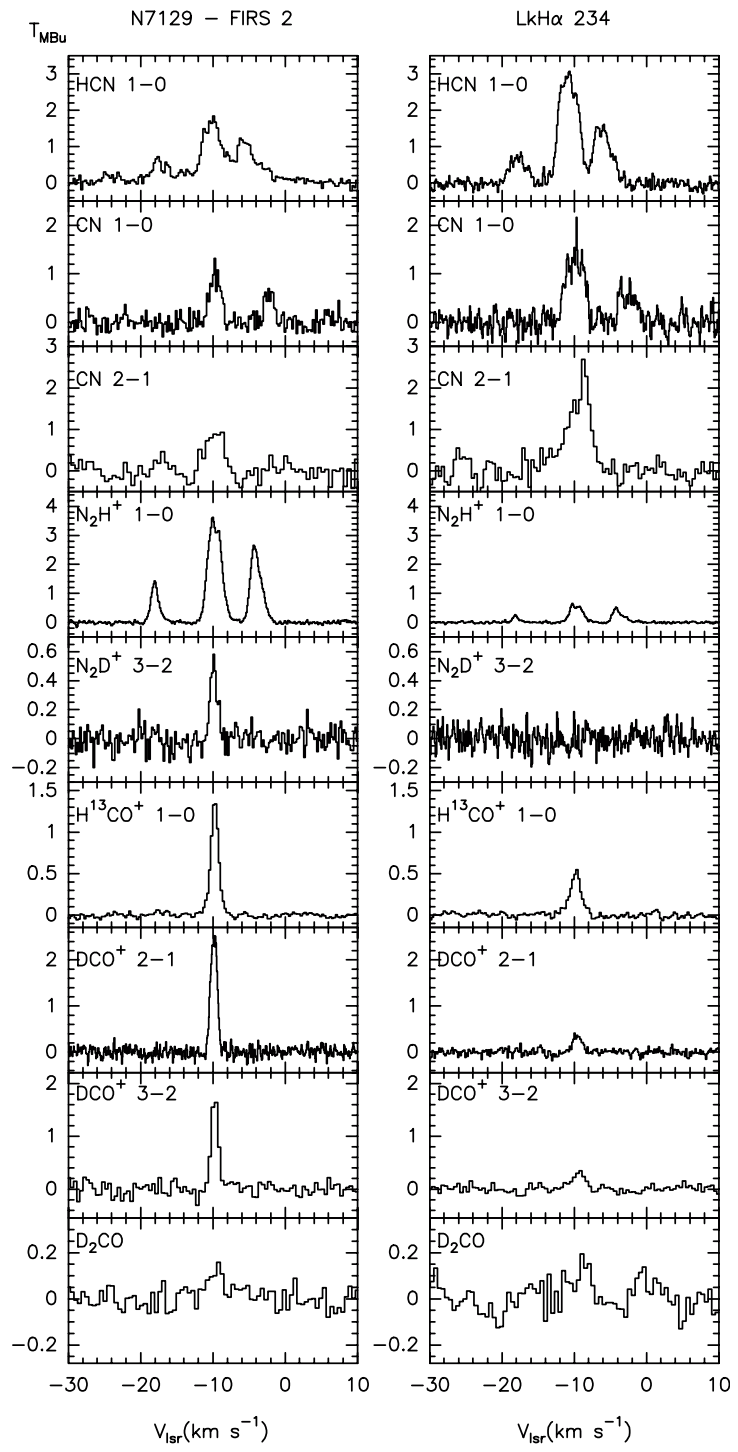

Fig. 15. Observed spectra towards the $(0,0)$ position in $\mathrm{LkH} \alpha 234$ and NGC 7129 - FIRS 2. The warming of the gas during the protostellar evolution produces significant chemical changes, especially in the cold component.

(see Fig. 15) show that, in contrast to the $\mathrm{CN} 1 \rightarrow 0$ line, the emission of the HCN $1 \rightarrow 0$ line comprises a significant contribution from the molecular outflow giving rise to this peculiar morphology.

The radicals $\mathrm{CN}$ and $\mathrm{HCN}$ are known to be especially abundant in PDRs. In particular, the $\mathrm{CN} / \mathrm{HCN}$ ratio has been successfully used as a PDR tracer in different kinds of object. In Fig. 16 we show the maps of the $(\mathrm{CN} 1 \rightarrow 0) /(\mathrm{HCN} 1 \rightarrow$ $0)$ intensity ratio in FIRS 2 and $\mathrm{LkH} \alpha$ 234. The $(\mathrm{CN} 1 \rightarrow$ $0) /(\mathrm{HCN} 1 \rightarrow 0)$ line intensity ratio is maximum at the star position and to the north, forming a conical feature with the star at its apex. We estimated the $\mathrm{CN}$ rotation temperature from the $(\mathrm{CN} 2 \rightarrow 1) /(\mathrm{CN} 1 \rightarrow 0)$ line intensity ratio (see Table 4$)$. Assuming the LTE approximation and the same rotation temperature for $\mathrm{CN}$ and $\mathrm{HCN}$, we obtain a $\mathrm{CN} / \mathrm{HCN}$ abundance ratio of $\sim 3$ at the star position. This value is similar to those found in PDRs and suggests that the gas chemistry in this conical feature is affected by the UV radiation from the protostar.

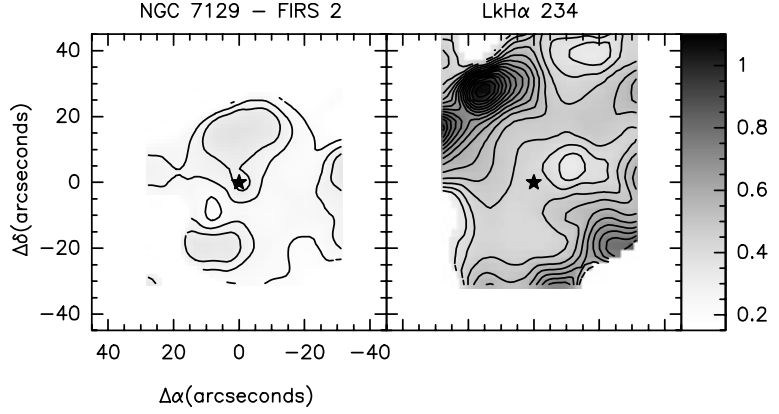

Fig. 16. $\mathrm{CN} 1 \rightarrow 0 / \mathrm{HCN} 1 \rightarrow 0$ integrated intensity ratio in NGC 7129 - FIRS 2 (left) and LkH $\alpha 234$ (right). Contour levels are 0.10 to 0.35 by 0.05 in NGC 7129 - FIRS 2 and 0.35 to 1.1 by 0.05 in $\mathrm{LkH} \alpha 234$.

However, the axis of this conical feature seems to be more similar to that of the outflow FIRS 2-out 2 than to that of the outflow FIRS 2-out 1 . This suggests that the PDR traced by the high $\mathrm{CN} / \mathrm{HCN}$ ratio could be related to the star driving the outflow FIRS 2-out 2 instead of to the Class 0 object. In fact, the PDR could be formed in the walls of the cavity excavated by the outflow FIRS 2-out 2 when they are illuminated by the exciting star. But observations with higher spatial resolution are required to reach a conclusion about this point.

We have also observed the $\mathrm{CN}$ and $\mathrm{HCN}$ lines towards $\mathrm{LkH} \alpha 234$. In this case the linewidths of the $\mathrm{CN}$ and $\mathrm{HCN}$ lines are similar, and in agreement with those found in the warm component. But, contrary to most of the observed molecular species, the $\mathrm{CN}$ and HCN emission do not peak at the star position but to the north, forming a conical feature. We have calculated the $\mathrm{CN} / \mathrm{HCN}$ integrated intensity ratio in the region. Surprisingly, the $\mathrm{CN} / \mathrm{HCN}$ ratio is minimum at the star position and maximum at the border of the clump as traced by the $1.3 \mathrm{~mm}$ observations, suggesting that the clump is illuminated from outside. Making column densities estimates, we derive $N(\mathrm{CN}) / N(\mathrm{HCN}) \sim 3$ at the star position.

Thus, the $\mathrm{CN} / \mathrm{HCN}$ fractional abundance ratio at the star position is equal (within the uncertainties) in FIRS 2 and $\mathrm{LkH} \alpha 234$ and consistent with the expected value in a PDR. However the behavior of the $\mathrm{CN} / \mathrm{HCN}$ ratio is very different in the rest of the envelope. In the case of FIRS 2, the $\mathrm{CN} / \mathrm{HCN}$ ratio decreases outwards from the star, as expected from a PDR illuminated from the interior and with an optically thick envelope. In the case of $\mathrm{LkH} \alpha 234$ the $\mathrm{CN} / \mathrm{HCN}$ ratio is quite constant inside the clump and increases at the edges. This suggests that the clump is also illuminated from outside (the clump is located on the border of an HII region). Since the envelope is less massive than that associated with FIRS 2, the whole envelope can be considered as a PDR.

\section{Deuterated compounds}

In order to derive the deuterium fractionation we observed the $\mathrm{DCO}^{+} 2 \rightarrow 1$ and $3 \rightarrow 2, \mathrm{~N}_{2} \mathrm{D}^{+} 3 \rightarrow 2$, and $\mathrm{D}_{2} \mathrm{CO} 4_{04} \rightarrow$ $3_{03}$ lines toward the studied regions. In Fig. 15 we show the spectra of these lines toward FIRS 2 and $\mathrm{LkH} \alpha 234$. 
Table 9. Beam-averaged column densities.

\begin{tabular}{|c|c|c|}
\hline $\begin{array}{l}\text { Age } \\
n\left(\mathrm{~cm}^{-3}\right)\end{array}$ & $\begin{array}{c}\text { NGC } 7129-\text { FIRS } 2 \\
\gtrsim 3000 \mathrm{yr} \\
5 \times 10^{5}-3 \times 10^{6}\end{array}$ & $\begin{array}{c}\mathrm{LkH} \alpha 234 \\
\sim 10^{5} \mathrm{yr} \\
2 \times 10^{5}\end{array}$ \\
\hline \multicolumn{3}{|c|}{ Cold envelope } \\
\hline$T_{k}$ & $13 \mathrm{~K}$ & $28 \mathrm{~K}$ \\
\hline$\Omega_{s}$ & $\sim 21 "$ & $\sim 8^{\prime \prime}$ \\
\hline $\mathrm{NH}_{3}$ & $4.9 \times 10^{14}$ & $4.0 \times 10^{13}$ \\
\hline $\mathrm{N}_{2} \mathrm{H}^{+}$ & $3.8 \times 10^{13}$ & $1.0 \times 10^{13}$ \\
\hline $\mathrm{H}^{13} \mathrm{CO}^{+}$ & $2.2 \times 10^{12}$ & $1.7 \times 10^{12}$ \\
\hline $\mathrm{DCO}^{+}$ & $1.8 \times 10^{12}$ & $4.2 \times 10^{11}$ \\
\hline $\mathrm{N}_{2} \mathrm{D}^{+}$ & $5.4 \times 10^{11}$ & $<2.3 \times 10^{11}$ \\
\hline $\mathrm{N}_{2} \mathrm{H}^{+} / \mathrm{H}^{13} \mathrm{CO}^{+}$ & 17 & 6 \\
\hline $\mathrm{NH}_{3} / \mathrm{N}_{2} \mathrm{H}^{+}$ & $13-33^{b}$ & 10 \\
\hline $\mathrm{DCO}^{+} / \mathrm{H}^{13} \mathrm{CO}^{+}$ & 0.7 & 0.2 \\
\hline $\mathrm{N}_{2} \mathrm{D}^{+} / \mathrm{N}_{2} \mathrm{H}^{+}$ & 0.014 & $<0.02$ \\
\hline \multicolumn{3}{|c|}{ Warm envelope } \\
\hline$T_{k}$ & $>50 \mathrm{~K}$ & $>100 \mathrm{~K}$ \\
\hline$\Omega_{s}$ & $\sim 9^{\prime \prime}$ & $\sim 14^{\prime \prime}$ \\
\hline $\mathrm{C}^{34} \mathrm{~S}$ & $1.5 \times 10^{12}$ & $1.7 \times 10^{12}$ \\
\hline $\mathrm{CH}_{3} \mathrm{OH}$ & $9.8 \times 10^{14}$ & $5.4 \times 10^{14}$ \\
\hline $\mathrm{CH}_{3} \mathrm{CN}$ & $3.6 \times 10^{12}$ & $>2.0 \times 10^{12}$ \\
\hline $\mathrm{H}_{2} \mathrm{CO}$ & $7.0 \times 10^{13}$ & $8.0 \times 10^{13}$ \\
\hline $\mathrm{H}_{2}{ }^{13} \mathrm{CO}$ & $2.3 \times 10^{12}$ & $2.4 \times 10^{12}$ \\
\hline $\mathrm{D}_{2} \mathrm{CO}$ & $2.0 \times 10^{12}$ & $3.7 \times 10^{12}$ \\
\hline $\mathrm{D}_{2} \mathrm{CO} / \mathrm{H}_{2} \mathrm{CO}$ & 0.03 & 0.05 \\
\hline \multicolumn{3}{|c|}{ PDR } \\
\hline $\mathrm{CN}$ & $4.8 \times 10^{13}$ & $6.1 \times 10^{13}$ \\
\hline $\mathrm{HCN}$ & $1.6 \times 10^{13}$ & $2.1 \times 10^{13}$ \\
\hline \multicolumn{3}{|c|}{ Outflow } \\
\hline $\mathrm{SiO}$ & $2.8 \times 10^{12}$ & $2.0 \times 10^{11}$ \\
\hline
\end{tabular}

${ }^{b}$ Assuming beam filling factor $\sim 1$ and a source size of $21^{\prime \prime}$ for the $\mathrm{NH}_{3}$ emitting region.

The integrated line intensity maps of the $\mathrm{DCO}^{+} 2 \rightarrow 1$ line toward FIRS 2 are shown in Fig. 4.

The linewidths of the $\mathrm{DCO}^{+}$and $\mathrm{N}_{2} \mathrm{D}^{+}$lines are $\sim 1.0 \mathrm{~km} \mathrm{~s}^{-1}$ which suggests that they arise in the cold component of the envelope like the non-deuterated compounds $\mathrm{HCO}^{+}$ and $\mathrm{N}_{2} \mathrm{H}^{+}$. The linewidth of the $\mathrm{D}_{2} \mathrm{CO}$ line is $\sim 4 \mathrm{~km} \mathrm{~s}^{-1}$ like those of the lines arising in the warm component, and in particular the lines of the chemically related species $\mathrm{H}_{2} \mathrm{CO}$ and $\mathrm{H}_{2}{ }^{13} \mathrm{CO}$. In Table 9 we show the $\mathrm{DCO}^{+} / \mathrm{H}^{13} \mathrm{CO}^{+}, \mathrm{N}_{2} \mathrm{D}^{+} / \mathrm{N}_{2} \mathrm{H}^{+}$ and $\mathrm{D}_{2} \mathrm{CO} / \mathrm{H}_{2} \mathrm{CO}$ abundance ratios in both sources. The $\mathrm{DCO}^{+} / \mathrm{H}^{13} \mathrm{CO}^{+}$abundance ratio is a factor of 20 lower in $\mathrm{LkH} \alpha 234$ than in FIRS 2. This factor is so large that it cannot be due to the $\mathrm{H}^{13} \mathrm{CO}^{+}$depletion but must instead be due to a different value of the deuterium fractionation in these cold envelopes. Thus, we propose that the deuterium fractionation in the cold envelope decreases during the protostellar evolution. As we will discuss in detail in the next section, this increase in the deuterium fractionation can be understood as the consequence of the envelope warming during the protostellar evolution.

A very different case is the $\mathrm{D}_{2} \mathrm{CO} / \mathrm{H}_{2} \mathrm{CO}$ abundance ratio, which increases by a factor $\sim 1.5$ from FIRS 2 to $\mathrm{LkH} \alpha 234$. Since a factor of 1.5 is within the uncertainties of our column density estimates, we conclude that the deuterium fractionation, as measured by the $\mathrm{D}_{2} \mathrm{CO} / \mathrm{H}_{2} \mathrm{CO}$ abundance ratio, seems to be constant (or slightly increase) in the warm component during the protostellar evolution. Thus, the evolution of the deuterium fractionation in the warm envelope seems to follow a different trend than in the cold envelope. The evaporation of the icy grain mantles is very likely the main process responsible of this behavior.

\section{Discussion}

\subsection{Physical structure of the YSOs envelopes and its evolution}

Our data show the existence of at least two well differentiated components in the envelope of FIRS 2 and $\mathrm{LkH} \alpha 234$, a cold envelope traced by the low energy lines of $\mathrm{NH}_{3}, \mathrm{~N}_{2} \mathrm{H}^{+}$ and $\mathrm{H}^{13} \mathrm{CO}^{+}$, and a warm envelope traced by the $\mathrm{CS}, \mathrm{CH}_{3} \mathrm{OH}$ and $\mathrm{H}_{2} \mathrm{CO}$ lines. These two components can be differentiated observationally by their kinematics, the morphology of their emission and by their physical conditions. Thus the lines arising in the cold component are narrow $\left(\Delta V \sim 1 \mathrm{~km} \mathrm{~s}^{-1}\right)$ and the emission peak is located at the star position in FIRS 2. Besides, the kinetic temperature of this gas estimated from the $\mathrm{NH}_{3}$ lines is $\sim 13 \mathrm{~K}$ in FIRS 2 and $\sim 28 \mathrm{~K}$ in $\mathrm{LkH} \alpha 234$.

A warm envelope component is detected towards these sources traced by the emission of species like $\mathrm{CS}, \mathrm{CH}_{3} \mathrm{OH}$ and $\mathrm{H}_{2} \mathrm{CO}$. These species present enhanced abundances in regions where the icy grain mantles are evaporated (van der Tak et al. 2000). They are also abundant in molecular outflows where they can be released into the gas phase by shock fronts. In FIRS 2, the emission of the low energy transitions of these species arises mainly in the bipolar outflow. However, this association is not clear in $\mathrm{LkH} \alpha 234$ where their emission could arise in the inner and warmer part of the envelope.

Finally, we have strong evidence for the existence of a hot core in the Class 0 protostar FIRS 2. The high density measured at ambient velocities from the $\mathrm{C}^{34} \mathrm{~S}$ lines, the high temperature component of the $\mathrm{CH}_{3} \mathrm{OH}$ lines and, above all, the detection of the $\mathrm{CH}_{3} \mathrm{CN}$ lines with a rotation temperature of $\sim 63 \mathrm{~K}$ show the existence of a hot core in this target.

\subsection{Physical and chemical evolution of the YSOs envelopes}

\subsubsection{Cold envelope}

In Table 9 we show the physical parameters and molecular column densities in the Class 0 protostar FIRS 2 and $\mathrm{LkH} \alpha 234$. The molecules $\mathrm{NH}_{3}, \mathrm{~N}_{2} \mathrm{H}^{+}, \mathrm{H}^{13} \mathrm{CO}^{+}$and their deuterated compounds $\mathrm{DCO}^{+}$and $\mathrm{N}_{2} \mathrm{D}^{+}$trace the cold envelope component. The column densities of these species decrease by a factor of 5-10 from the Class 0 protostar to the Type I Herbig Be star, showing that the mass of the cold envelope decreases by at least a factor of 5 during the protostellar phase. Based on the $\mathrm{NH}_{3}$ and $\mathrm{N}_{2} \mathrm{H}^{+}$data we have also derived the kinetic temperature and size of this cold component. The kinetic temperature increases from $\sim 13$ to $\sim 28 \mathrm{~K}$ and the size of the emitting region decreases from 21" to 6" from FIRS 2 and $\mathrm{LkH} \alpha 234$. This is 
consistent with previous results by Fuente et al. (2002) which shows that the protostellar envelope is dispersed and becomes warmer during the evolution of the protostar into a visible star.

The warming of the cold envelope produces changes in its chemical composition. In Table 5 we show the $\mathrm{N}_{2} \mathrm{H}^{+} / \mathrm{H}^{13} \mathrm{CO}^{+}$ and $\mathrm{NH}_{3} / \mathrm{N}_{2} \mathrm{H}^{+}$abundance ratios in several positions. Note that the $\mathrm{N}_{2} \mathrm{H}^{+} / \mathrm{H}^{13} \mathrm{CO}^{+}$ratio is different in the studied objects. The $\mathrm{N}_{2} \mathrm{H}^{+} / \mathrm{H}^{13} \mathrm{CO}^{+}$ratio is $\sim 17$ in the cold young object FIRS 2 and $\sim 6$ in the more evolved and warmer Herbig Be star $\mathrm{LkH} \alpha 234$. As discussed in Sect. 3.2, this gradient in the $\mathrm{N}_{2} \mathrm{H}^{+} / \mathrm{H}^{13} \mathrm{CO}^{+}$ ratio is very likely due to the $\mathrm{H}^{13} \mathrm{CO}^{+}$depletion in the cold envelope of the protostar. Molecular depletion is expected to be significant only for kinetic temperatures $T_{k}<20 \mathrm{~K}$. Thus, molecular depletion is negligible in the envelope of $\mathrm{LkH} \alpha 234$ where the gas kinetic temperature is $\sim 28 \mathrm{~K}$.

Within the nitrogen chemistry, we have also studied the $\mathrm{NH}_{3} / \mathrm{N}_{2} \mathrm{H}^{+}$ratio. Since the beam is very different for the $\mathrm{NH}_{3}$ and $\mathrm{N}_{2} \mathrm{H}^{+}$observations, this abundance ratio is very dependent on the assumed source size. For $\mathrm{LkH} \alpha 234$ we have been able to calculate both, the $\mathrm{NH}_{3}$ and $\mathrm{N}_{2} \mathrm{H}^{+}$emitting region size. We derived the same size, $\sim 6^{\prime \prime}-8^{\prime \prime}$, for both molecules and the $\mathrm{NH}_{3} / \mathrm{N}_{2} \mathrm{H}^{+}$ratio is $\sim 10$. In the case of FIRS 2 we have not been able to derive the source size from the $\mathrm{NH}_{3}$ emission but we have estimated a size of $\sim 21^{\prime \prime}$ from the $\mathrm{N}_{2} \mathrm{H}^{+}$observations. We assumed two limiting cases for the calculations of the $\mathrm{NH}_{3} / \mathrm{N}_{2} \mathrm{H}^{+}$ratio in this source. Assuming a beam filling factor of $\sim 1$ for both molecules we obtain $\mathrm{NH}_{3} / \mathrm{N}_{2} \mathrm{H}^{+} \sim 13$ in this object. However if we assume that the size of the $\mathrm{NH}_{3}$ emission is $\sim 21^{\prime \prime}$, like in the case of $\mathrm{N}_{2} \mathrm{H}^{+}$, we obtain $\mathrm{NH}_{3} / \mathrm{N}_{2} \mathrm{H}^{+} \sim 33$. In this case, we would have an $\mathrm{NH}_{3}$ abundance enhancement in the colder envelope of the Class 0 protostar. Recent results in pre-stellar cores show that the $\mathrm{NH}_{3}$ abundance could be enhanced in dense regions of these cores where the $\mathrm{CO}$ is expected to be depleted (Tafalla et al. 2004).

\subsubsection{Warm envelope}

When the star heats the envelope a sublimation front proceeds outwards from the star and removes molecules from grain mantles. The region of the envelope in which the gas kinetic temperature is high enough to evaporate the grain mantles is what we have called the "warm envelope". The species released to the gas phase are called "parent molecules" and their abundances increase significantly. The molecules $\mathrm{CH}_{3} \mathrm{OH}, \mathrm{NH}_{3}$, and $\mathrm{H}_{2} \mathrm{CO}$ are in this group. These molecules drive a high temperature chemistry giving rise to "daughter" molecules like $\mathrm{CH}_{3} \mathrm{CN}$. Within this scheme, $\mathrm{CH}_{3} \mathrm{OH}, \mathrm{H}_{2} \mathrm{CO}$ and $\mathrm{CH}_{3} \mathrm{CN}$ are tracers of the warm part of the envelope where the ices have been evaporated (Rodgers \& Charnley 2003). Some of these species are also abundant in the molecular outflow where shock fronts remove them from the grain mantles, and to a lesser extent in the cold envelope. This is the case for $\mathrm{NH}_{3}$ with the emission in the cold envelope dominated by the low-lying transitions in both, FIRS 2 and $\mathrm{LkH} \alpha$ 234, while the $(3,3)$ line arises in the warmer component.

In Table 9 we show the physical conditions and the molecular column densities in the warm envelopes of FIRS 2 and

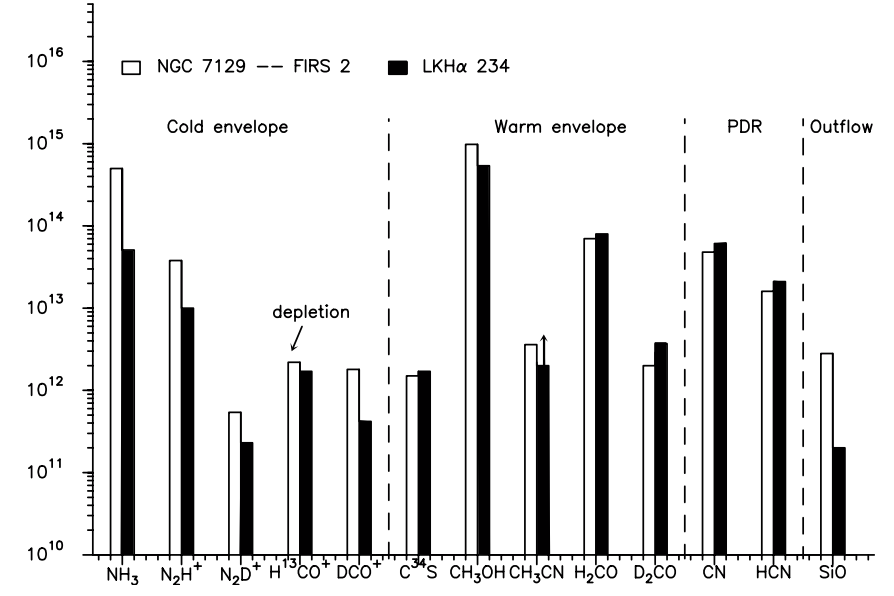

Fig. 17. Histogram with the total beam-averaged molecular column densities estimated in the IM Class 0 YSO NGC 7129-FIRS 2 and the $\mathrm{HBe}$ star LkH $\alpha 234$. The column densities of the molecules tracing the cold envelope decrease by at least a factor of 5 between NGC 7129 FIRS 2 and $\mathrm{LkH} \alpha 234$. An exception is $\mathrm{H}^{13} \mathrm{CO}^{+}$, which is depleted in the Class 0 protostar (see text). The column densities of the molecules tracing the warm envelope and the PDR remain quite constant, and in some cases increase, from the Class 0 protostar to the $\mathrm{HBe}$ star. The SiO column density is lower by almost 2 orders of magnitude in LkH $\alpha 234$ than in NGC 7129 - FIRS 2. This is very likely related to the absence of an energetic bipolar outflow in the HBe star.

$\mathrm{LkH} \alpha 234$. Contrary to the species tracing the cold envelopes, the species tracing the warm envelopes present similar column densities in both targets (see Fig. 17). In fact, some species like $\mathrm{H}_{2} \mathrm{CO}$ and $\mathrm{C}^{34} \mathrm{~S}$ seem to have larger column densities in $\mathrm{LkH} \alpha 234$. On the other hand, the size of the warm envelope is larger in the case of the Herbig Be star than in the case of the Class 0 YSO. Thus, the mass and size of the warm envelope remain quite constant, or even increase, during the protostellar evolution.

Although the column densities of the "warm envelope" species are not very different in the two YSOs, the origin of their emission could be different. The $\mathrm{CH}_{3} \mathrm{OH}$ and $\mathrm{H}_{2} \mathrm{CO}$ emission seem to be dominated by the molecular outflow in FIRS 2 . In the case of $\mathrm{CH}_{3} \mathrm{OH}$ we have detected two components in FIRS 2. The one centered at $\sim 7 \mathrm{~km} \mathrm{~s}^{-1}$ is clearly associated with the molecular outflow FIRS 2-out 1 and dominates the $\mathrm{CH}_{3} \mathrm{OH}$ emission in all the transitions with $E_{u}<100 \mathrm{~K}$. We observed only two low-energy $\mathrm{H}_{2} \mathrm{CO}$ lines in our targets. The emission of these lines in FIRS 2 is clearly associated with the outflow FIRS 2-out 1 (see Fig. 13).

Intense $\mathrm{CH}_{3} \mathrm{OH}$ and $\mathrm{H}_{2} \mathrm{CO}$ lines have also been detected in $\mathrm{LkH} \alpha 234$. In this case the link between these species and the molecular outflow is not clear. In fact, these lines could arise from a warm inner envelope where the icy mantles are being evaporated, releasing these species to the gas phase. Thus, although the $\mathrm{CH}_{3} \mathrm{OH}$ and $\mathrm{H}_{2} \mathrm{CO}$ column densities are very similar in both objects, the mechanism which removes these species from the icy mantles can be different in the Class 0 protostar FIRS 2 and the Herbig Be star LkH $\alpha$ 234. While shock fronts are very likely the main mechanism for the erosion of grain 
mantles in FIRS 2, ice evaporation could be a dominant mechanism in $\mathrm{LkH} \alpha 234$.

\subsection{3. $\mathrm{CN}, \mathrm{HCN}$}

The $\mathrm{CN}$ and HCN column densities differ in FIRS 2 and in $\mathrm{LkH} \alpha 234$ by a factor of $\sim 6$. This is easily understood within an evolutionary trend. Since the cold envelope has already been dispersed in $\mathrm{LkH} \alpha 234$, the UV radiation can penetrate deeper into the cloud affecting the chemistry throughout the envelope in $\mathrm{LkH} \alpha$ 234. The enhancement of the ionization fraction (in particular, the enhancement in the $\mathrm{C}^{+}$abundance) in the $\mathrm{LkH} \alpha 234$ envelope could produce an enhancement in the fractional abundances of the nitrogenated chains $\mathrm{CN}$ and $\mathrm{HCN}$ relative to $\mathrm{N}_{2} \mathrm{H}^{+}$and $\mathrm{NH}_{3}$.

The $\mathrm{CN} / \mathrm{HCN}$ ratio has been widely used as a PDR tracer. Values of the $\mathrm{CN} / \mathrm{HCN}$ ratio $>1$ have been considered proof of the existence of a photon-dominated chemistry region (see e.g. Fuente et al. 1993, 1995, 2003; Bachiller et al. 1997; Rodríguez-Franco et al. 1998). We detected a small region in the vicinity of the Class 0 source in FIRS 2 in which the $\mathrm{CN} / \mathrm{HCN}$ ratio is $>1$. This strongly suggests the existence of an incipient PDR in the inner part of this protostellar envelope. However, our limited angular resolution prevents us from discerning if this PDR is associated with the Class 0 source or with the IR companion star which is driving the outflow FIRS 2out 2 .

\subsubsection{Deuterated compounds}

We observed $\mathrm{DCO}^{+}$and $\mathrm{N}_{2} \mathrm{D}^{+}$in order to be able to derive the $\mathrm{D} / \mathrm{H}$ ratio in the cold envelopes of these stars and its possible changes during the evolution of the central object. Enhancements of the $\mathrm{D} / \mathrm{H}$ ratio over the $10^{-5}$ ratio of $\mathrm{HD} / \mathrm{H}_{2}$ have been found in dark clouds and young protostars (e.g. Butner et al. 1995; Williams et al. 1998). There are two main ways of producing these enhancements. Firstly, grain surface chemistry may enhance molecular $\mathrm{D} / \mathrm{H}$ ratios (Tielens 1983; Brown \& Millar 1989a,b; Charnley et al. 1997). Secondly, some key gas phase reactions involving destruction of deuterated species run more slowly at low temperatures than the equivalent reactions with hydrogen, and this leads to molecular D/H enhancement where a cold gas phase chemistry has been active (Roberts \& Millar 2000a,b). Furthermore, in colder gas, depletion of heavy molecules such as $\mathrm{CO}$ results in an increase of $\mathrm{H}_{2} \mathrm{D}^{+} / \mathrm{H}_{3}^{+}$and molecular $\mathrm{D} / \mathrm{H}$ ratios (Dalgarno \& Lepp 1984; Brown \& Millar 1989a; Roberts \& Millar 2000a,b). We found $\mathrm{DCO}^{+} / \mathrm{H}^{13} \mathrm{CO}^{+} \sim 0.7$ in FIRS 2 and $\sim 0.25$ in $\mathrm{LkH} \alpha 234$. Assuming a ${ }^{12} \mathrm{C} /{ }^{13} \mathrm{C}$ isotopic ratio of 89 , this would imply $\mathrm{DCO}^{+} / \mathrm{HCO}^{+} \sim 0.008$ in FIRS 2 and $\sim 0.003$ in $\mathrm{LkH} \alpha 234$. These ratios are similar to those found in the low mass Class 0 protostar IRAS $+16293-2422$ by van Dishoeck et al. (1995) but are lower than the values found in dark clouds (see e.g. Tiné et al. 2000). A cold gas chemistry as well as depletion of heavy molecules can explain the enhanced values of the $\mathrm{DCO}^{+} / \mathrm{HCO}^{+}$ratio found in the young stellar object FIRS 2 .
We also observed the doubly deuterated formaldehyde $\mathrm{D}_{2} \mathrm{CO}$. The linewidths of the observed $\mathrm{D}_{2} \mathrm{CO}$ lines show that this species, like the non-deuterated compound $\mathrm{H}_{2} \mathrm{CO}$, arises in the warm envelope. The $\mathrm{D}_{2} \mathrm{CO}$ column densities as well as the $\mathrm{D}_{2} \mathrm{CO} / \mathrm{H}_{2} \mathrm{CO}$ ratio are similar in $\mathrm{LkH} \alpha 234$ to those in FIRS 2. This suggests that the deuterium fractionation remains constant (or even increases, see Table 9) in the warm envelope during the protostellar evolution. Thus, the evolution of the deuterium fractionation is different in the cold and warm part of the envelope. Grain-surface chemistry may enhance the deuterium fractionation in the warm envelope, where molecular evaporation is very likely the main chemical phenomenum, while the cold gas chemistry and depletion could determine the evolution of the deuterium fractionation in the cold envelope.

The deuterium fractionation has been proposed as a chemical clock in YSOs. Our data confirm that the deuterium fractionation changes significantly during the protostellar evolution and consequently, can be used as a chemical clock. However, these changes are different in the cold and in the warm part of the envelope, and the measured $\mathrm{D} / \mathrm{H}$ ratio is very dependent on the molecular compounds used to determine it.

\section{Summary and conclusions: Chemical clocks in intermediate-mass YSOs}

We have carried out a molecular survey towards the IM YSOs FIRS 2 and $\mathrm{LkH} \alpha$ 234. Our survey confirms that protostellar envelopes are very complex objects composed by several components characterized by different physical and chemical properties:

1. A cold envelope observationally characterized by narrow lines $\left(\Delta V \sim 1 \mathrm{~km} \mathrm{~s}^{-1}\right)$ and low kinetic temperatures $\left(T_{\mathrm{k}}<50 \mathrm{~K}\right)$. This component is well traced by the species $\mathrm{NH}_{3}, \mathrm{~N}_{2} \mathrm{H}^{+}, \mathrm{H}^{13} \mathrm{CO}^{+}, \mathrm{DCO}^{+}$and $\mathrm{N}_{2} \mathrm{D}^{+}$.

2. A warm envelope observationally characterized by large linewidths $\left(\Delta V>3 \mathrm{~km} \mathrm{~s}^{-1}\right)$ and higher kinetic temperatures $\left(T_{\mathrm{k}}>50 \mathrm{~K}\right)$. The low energy transitions of $\mathrm{CS}, \mathrm{C}^{34} \mathrm{~S}$, $\mathrm{CH}_{3} \mathrm{OH}$, and $\mathrm{H}_{2} \mathrm{CO}$ arise in this warm component.

3. A hot core characterized by high densities $\left(n>10^{6} \mathrm{~cm}^{-3}\right)$ and a high kinetic temperature $\left(T_{\mathrm{k}}>100 \mathrm{~K}\right)$. The symmetric rotor $\mathrm{CH}_{3} \mathrm{CN}$ seems to be the best tracer of this hot component.

4. In addition to these envelope components, energetic outflows are associated with very young stellar objects. In addition to $\mathrm{CO}$ and its isotopes, the $\mathrm{SiO}$ emission is very likely the best tracer of the outflow component for these objects.

Once we have used chemistry to determine the physical structure of the YSOs, FIRS 2 and $\mathrm{LkH} \alpha$ 234, we can determine the evolution of the protostellar envelopes of IM stars during the protostellar phase. FIRS 2 is an IM Class 0 object while $\mathrm{LkH} \alpha 234$ is a very young (and still deeply embedded) $\mathrm{HBe}$ star. As expected, different physical conditions and chemistry are found in these objects. The Class 0 IM is a cold object $\left(T_{\mathrm{k}} \sim 13 \mathrm{~K}\right)$ in which molecular depletion is still important. We have no evidence of a change in the $\mathrm{N}_{2} \mathrm{H}^{+}$abundance between 
Table 10. Chemical diagnostics for YSO evolution.

\begin{tabular}{lcc}
\hline \hline & NGC 7129-FIRS 2 & LkH $\alpha 234$ \\
\hline $\mathrm{SiO} / \mathrm{C}^{34} \mathrm{~S}$ & $\sim 2$ & $\sim 0.1$ \\
$\mathrm{CN} / \mathrm{N}_{2} \mathrm{H}^{+}$ & $\sim 1$ & $\sim 6$ \\
$\mathrm{HCN} / \mathrm{N}_{2} \mathrm{H}^{+}$ & $\sim 0.4$ & $\sim 2$ \\
$\mathrm{DCO}^{+} / \mathrm{HCO}^{+}$ & $\sim 0.008$ & $\sim 0.002$ \\
$\mathrm{D}_{2} \mathrm{CO} / \mathrm{DCO}^{+}$ & $\sim 1$ & $\sim 10$ \\
\hline
\end{tabular}

the two objects. The decrease in the $\mathrm{N}_{2} \mathrm{H}^{+}$column density observed in Fig. 17 is the consequence of the disruption of the cold envelope during the stellar evolution. However, we have found evidence for $\mathrm{H}^{13} \mathrm{CO}^{+}$depletion in FIRS 2. Molecules like $\mathrm{CH}_{3} \mathrm{OH}$ and $\mathrm{H}_{2} \mathrm{CO}$ are expected to trace mainly the warm region in which grain mantles have been evaporated. The column densities of these molecules remain constant (or increase) from Class 0 IM to the more evolved HBe star, suggesting that the abundances of these molecules and/or the mass of the warm gas increases with the protostellar evolution. The detection of $\mathrm{CH}_{3} \mathrm{CN}$ and the high temperature derived from it $\left(T_{\mathrm{k}} \sim 63 \mathrm{~K}\right)$ shows that a hot core has developed in the Class 0 protostar FIRS 2. Thus, our results suggest an evolutionary sequence in which as the protostar evolves to become a visible star, the total column density of gas decreases while the amount of warm gas remains quite constant or increases slightly. These physical and chemical changes imply important changes in the beamaveraged column densities during the protostellar evolution.

Based on our observational study of FIRS 2 and $\mathrm{LkH} \alpha 234$, we propose some abundance ratios that can be used as chemical clocks in YSOs. These ratios are defined to be useful tools to distinguish between different evolutionary stages of YSOs, but do not correspond to the actual abundance ratios in any of the envelope components. They have been calculated with beam-averaged column densities and, as largely discussed in this paper, are the consequence of complex physical and chemical evolution in the whole envelope.

In Table 10 we list the proposed chemical clocks. We find the maximum variation between the Class 0 and the $\mathrm{HBe}$ object when we compare the $\mathrm{SiO} / \mathrm{C}^{34} \mathrm{~S}$ ratio. This ratio decreases by a factor of $\sim 20$ between these two objects. This decrease is the consequence of the decay of the bipolar outflow phenomenum during the protostellar evolution. This ratio is especially useful to determine the evolutionary stage of the youngest objects, which are associated with the most energetic bipolar outflows. The nitrogen chemistry is also useful to determine the evolutionary stage of YSOs. The $\mathrm{CN} / \mathrm{N}_{2} \mathrm{H}^{+}$and $\mathrm{HCN} / \mathrm{N}_{2} \mathrm{H}^{+}$ratios are larger by a factor of $\sim 6$ in the $\mathrm{HBe}$ star than in the Class 0 object. As commented above this is mainly due to the fact that the $\mathrm{LkH} \alpha 234$ envelope is thinner and warmer than that of the FIRS 2. These ratios would probably be more useful for differentiating between objects in late protostellar evolution when the protostellar envelope becomes optically thinner. Finally, the deuterated species could also be a good indicator of the protostellar evolution. The $\mathrm{DCO}^{+} / \mathrm{HCO}^{+}$ratio decreases by a factor $\sim 4$ because of the warmer envelope in $\mathrm{LkH} \alpha 234$. However, we can have a different behavior in the deuterated species whose emission arises in the warm envelope component. This is the case of the doubly deuterated compound $\mathrm{D}_{2} \mathrm{CO}$. We obtain the largest contrast in the abundance ratio if we compare the $\mathrm{D}_{2} \mathrm{CO}$ and $\mathrm{DCO}^{+}$abundances. The $\mathrm{D}_{2} \mathrm{CO} / \mathrm{DCO}^{+}$ratio increases by a factor of 10 from the Class 0 to the Herbig Be star. However, we should be cautious in using this ratio because we are comparing species arising in different regions of the envelope.

Acknowledgements. We are grateful to the MPIfR and IRAM staff in Pico de Veleta for their support during the observations. This work has been partially supported by the Spanish DGICYT under grant AYA2003-07584 and Spanish DGI/SEPCT under grant ESP200304957. J.R.R. acknowledges the financial support from AYA200306473.

\section{References}

André, P., Ward-Thompson, D., \& Barsony, M. 2000, Protostars and Planets IV, 59

Bachiller, R., Guilloteau, S., \& Kahane, C. 1987, A\&A, 173, 324

Bachiller, R., Martín-Pintado, J., \& Fuente, A. 1991, A\&A, 243, L21

Bachiller, R. 1996, Molecules in Astrophysics: Probes \& Processes, IAU Symp., 178, 103

Bachiller, R., Fuente, A., Bujarrabal, V., et al. 1997, A\&A, 319, 235

Bachiller, R., Pérez Gutiérrez, M., Kumar, M. S. N., \& Tafalla, M. 2001, A\&A, 372, 899

Bottinelli, S., Ceccarelli, C., Lefloch, B., et al., 2004, ApJL, in press [arXiv: astro-ph/0410601]

Brown, P. D., Charnley, S. B., \& Millar, T. J. 1988, MNRAS, 231, 409

Brown, P. D., \& Millar, T. J. 1989a, MNRAS, 240, 25

Brown, P. D., \& Millar, T. J. 1989b, MNRAS, 237, 661

Butner, H. M., Lada, E. A., \& Loren, R. B. 1995, ApJ, 448, 207

Caselli, P., Hasegawa, T. I., \& Herbst, E. 1993, ApJ, 408, 548

Caselli, P., Walmsley, C. M., Tafalla, M., Dore, L., \& Myers, P. C. 1999, ApJ, 523, L165

Caselli, P., Walmsley, C. M., Zucconi, A., et al. 2002, ApJ, 565, 344

Cazaux, S., Tielens, A. G. G. M., Ceccarelli, C., et al. 2003, ApJ, 593, L51

Charnley, S. B., Tielens, A. G. G. M., \& Millar, T. J. 1992, ApJ, 399, L71

Charnley, S. B., Tielens, A. G. G. M., \& Rodgers, S. D. 1997, ApJ, 482, L203

Codella, C., Bachiller, R., \& Reipurth, B. 1999, A\&A, 343, 585

Dalgarno, A., \& Lepp, S. 1984, ApJ, 287, L47

Danby, G., Flower, D. R., Valiron, P., Schilke, P., \& Walmsley, C. M. 1988, MNRAS, 235, 229

Eiroa, C., Palacios, J., \& Casali, M. M. 1998, A\&A, 335, 243

Flower, D. R., Pineau des Forets, G., Field, D., \& May, P. W. 1996, MNRAS, 280, 447

Fuente, A., Martin-Pintado, J., Cernicharo, J., \& Bachiller, R. 1993, A\&A, 276, 473

Fuente, A., Martin-Pintado, J., \& Gaume, R. 1995, ApJ, 442, L33

Fuente, A., Martín-Pintado, J., Bachiller, R., Neri, R., \& Palla, F. 1998, A\&A, 334, 253

Fuente, A., Neri, R., Martín-Pintado, J., et al. 2001, A\&A, 366, 873

Fuente, A., Martín-Pintado, J., Bachiller, R., Rodríguez-Franco, A., \& Palla, F. 2002, A\&A, 387, 977

Fuente, A., Rodríguez-Franco, A., García-Burillo, S., Martín-Pintado, J., \& Black, J. H. 2003, A\&A, 406, 899

García-Burillo, S., Martín-Pintado, J., Fuente, A., \& Neri, R. 2001, ApJ, 563, L27

García-Burillo, S., Martín-Pintado, J., Fuente, A., \& Neri, R. 2000, A\&A, 355, 499 
Green, S., \& Chapman, S. 1978, ApJS, 37, 169

Gueth, F., Guilloteau, S., \& Bachiller, R. 1996, A\&A, 307, 891

Lee, J.-E., Evans, N. J., \& Shirley, Y. L. 2003, ApJ, 583, 789

Jørgensen, J. K., Hogerheijde, M. R., van Dishoeck, E. F., Blake,

G. A., \& Schöier, F. L. 2004a, A\&A, 413, 993

Jørgensen, J. K., Schöier, F. L., \& van Dishoeck, E. F. 2004b, A\&A, 416, 603

Maret, S., Ceccarelli, C., Caux, E., et al. 2004, A\&A, 416, 577

Martín-Pintado, J., Bachiller, R., \& Fuente, A. 1992, A\&A, 254, 315

Nomura, H., \& Millar, T. J. 2004, A\&A, 414, 409

Pickett, H. M., Poynter, R. L., Cohen, E. A., et al. 1998, J. Quant. Spectrosc. \& Rad. Trans., 60, 883

Ray, T. P., Poetzel, R., Solf, J., \& Mundt, R., 1990, ApJ, 357, L45

Roberts, H., \& Millar, T. J. 2000a, A\&A, 361, 388

Roberts, H., \& Millar, T. J. 2000b, A\&A, 364, 780

Rodgers, S. D., \& Charnley, S. B. 2003, ApJ, 585, 355

Rodríguez-Franco, A., Martin-Pintado, J., \& Fuente, A. 1998, A\&A, 329, 1097

Schilke, P., Pineau des Forêts, G., Walmsley, C. M., \& Martín-Pintado, J. 2001, A\&A, 372, 291
Tafalla, M., Myers, P. C., Caselli, P., Walmsley, C. M., \& Comito, C. 2002, ApJ, 569, 815

Tafalla, M., Myers, P. C., Caselli, P., \& Walmsley, C. M. 2004, A\&A, 416, 191

Testi, L., Palla, F., \& Natta, A. 1999, A\&A, 342, 515

Tielens, A. G. G. M. 1983, A\&A, 119, 177

Tiné, S., Roueff, E., Falgarone, E., Gerin, M., \& Pineau des Forêts, G. 2000, A\&A, 356, 1039

van Dishoeck, E. F., Blake, G. A., Jansen, D. J., \& Groesbeck, T. D. 1995, ApJ, 447, 760

Van der Tak, F. F. S., van Dishoeck, E. F., \& Caselli, P. 2000, A\&A, 361,327

Viti, S., Collings, M. P., Dever, J. W., McCoustra, M. R. S., \& Williams, D.A. 2004, MNRAS, 354, 1141

Williams, J. P., Bergin, E. A., Caselli, P., Myers, P. C., \& Plume, R. 1998, ApJ, 503, 689

Wolfire, M. G., McKee, C. F., Hollenbach, D., \& Tielens, A. G. G. M. 2003, ApJ, 587, 278 
A. Fuente et al.: Chemical evolution in the environment of intermediate mass young stellar objects, Online Material p 1

\section{Online Material}


Table 1. Description of the IRAM 30 m observations.

\begin{tabular}{|c|c|c|c|c|}
\hline Line & Freq. (GHz) & $H P B W$ & $\eta_{\mathrm{MB}}$ & Observed positions \\
\hline $\mathrm{N}_{2} \mathrm{H}^{+} 1 \rightarrow 0$ & 93173.2 & $26.5^{\prime \prime}$ & 0.71 & $60^{\prime \prime} \times 60^{\prime \prime}$ map \\
\hline $\mathrm{CN} 1 \rightarrow 0$ & 113490.0 & $23^{\prime \prime}$ & 0.65 & $60^{\prime \prime} \times 60^{\prime \prime} \operatorname{map}\left(\right.$ FIRS 2), 60"× $\times 100^{\prime \prime} \operatorname{map}(\mathrm{LkH} \alpha 234)$ \\
\hline $\mathrm{HCN} 1 \rightarrow 0$ & 88631.8 & $27.5^{\prime \prime}$ & 0.73 & $60^{\prime \prime} \times 60^{\prime \prime} \operatorname{map}\left(\right.$ FIRS 2), $60 " \times 100^{\prime \prime} \operatorname{map}(\mathrm{LkH} \alpha 234)$ \\
\hline $\mathrm{H}^{13} \mathrm{CO}^{+} 1 \rightarrow 0$ & 86754.3 & $27.5^{\prime \prime}$ & 0.73 & complete map \\
\hline $\mathrm{HC}^{18} \mathrm{O}^{+} 1 \rightarrow 0$ & 85162.2 & $27.5^{\prime \prime}$ & 0.73 & $(0,0)$ \\
\hline $\mathrm{SiO} 2 \rightarrow 1$ & 86846.9 & $27.5^{\prime \prime}$ & 0.73 & complete map \\
\hline $\mathrm{SiO} 3 \rightarrow 2$ & 130268.6 & $20^{\prime \prime}$ & 0.60 & complete map \\
\hline $\mathrm{CS} 3 \rightarrow 2$ & 146969.0 & $16.5^{\prime \prime}$ & 0.55 & complete map \\
\hline $\mathrm{C}^{34} \mathrm{~S} 2 \rightarrow 1$ & 96412.9 & $25.9^{\prime \prime}$ & 0.70 & $(0,0)$ \\
\hline $\mathrm{C}^{34} \mathrm{~S} 3 \rightarrow 2$ & 144617.1 & $17^{\prime \prime}$ & 0.55 & $(0,0)$ \\
\hline$C^{34} S 5 \rightarrow 4$ & 192818.5 & $13^{\prime \prime}$ & 0.47 & $(0,0)$ \\
\hline $\mathrm{CH}_{3} \mathrm{OH} 2(1,3) \rightarrow 1(1,3)$ & 96755.5 & $26^{\prime \prime}$ & 0.70 & $72^{\prime \prime} \times 72^{\prime \prime}$ map \\
\hline $\mathrm{CH}_{3} \mathrm{OH} 2(0,3) \rightarrow 1(0,3)$ & 96744.6 & $26^{\prime \prime}$ & 0.70 & $72^{\prime \prime} \times 72^{\prime \prime}$ map \\
\hline $\mathrm{CH}_{3} \mathrm{OH} 2(1,4) \rightarrow 1(1,4)$ & 96741.4 & $26^{\prime \prime}$ & 0.70 & $72^{\prime \prime} \times 72^{\prime \prime}$ map \\
\hline $\mathrm{CH}_{3} \mathrm{OH} 2(0,1) \rightarrow 1(0,1)$ & 96739.4 & $26^{\prime \prime}$ & 0.70 & $72^{\prime \prime} \times 72^{\prime \prime}$ map \\
\hline $\mathrm{CH}_{3} \mathrm{OH} 3(0,3) \rightarrow 2(0,3)$ & 145093.7 & $17^{\prime \prime}$ & 0.55 & $(0,0)$ \\
\hline $\mathrm{CH}_{3} \mathrm{OH} 3(1,4) \rightarrow 2(1,4)$ & 145097.5 & $17^{\prime \prime}$ & 0.55 & $(0,0)$ \\
\hline $\mathrm{CH}_{3} \mathrm{OH} 3(0,1) \rightarrow 2(0,1)$ & 145103.2 & $17^{\prime \prime}$ & 0.55 & $(0,0)$ \\
\hline $\mathrm{CH}_{3} \mathrm{OH} 3(2,2) \rightarrow 2(2,2)^{a}$ & 145124.4 & $17^{\prime \prime}$ & 0.55 & $(0,0)$ \\
\hline $\mathrm{CH}_{3} \mathrm{OH} 3(1,3) \rightarrow 2(1,3)$ & 145131.9 & $17^{\prime \prime}$ & 0.55 & $(0,0)$ \\
\hline $\mathrm{CH}_{3} \mathrm{OH} 5(2,4) \rightarrow 4(2,4)$ & 241904.1 & $10^{\prime \prime}$ & 0.40 & $72^{\prime \prime} \times 72^{\prime \prime}$ map \\
\hline $\mathrm{CH}_{3} \mathrm{OH} 5(0,1) \rightarrow 4(0,1)$ & 241791.4 & $10^{\prime \prime}$ & 0.40 & $72^{\prime \prime} \times 72^{\prime \prime}$ map \\
\hline $\mathrm{CH}_{3} \mathrm{OH} 5(1,4) \rightarrow 4(1,4)$ & 241767.2 & $10^{\prime \prime}$ & 0.40 & $72^{\prime \prime} \times 72^{\prime \prime}$ map \\
\hline $\mathrm{CH}_{3} \mathrm{OH} 5(3,1) \rightarrow 4(3,1)$ & $241832.9^{a}$ & $10^{\prime \prime}$ & 0.40 & $72^{\prime \prime} \times 72^{\prime \prime}$ map \\
\hline $\mathrm{CH}_{3} \mathrm{OH} 5(2,2) \rightarrow 4(2,2)$ & 241842.3 & $10^{\prime \prime}$ & 0.40 & $72^{\prime \prime} \times 72^{\prime \prime}$ map \\
\hline $\mathrm{CH}_{3} \mathrm{OH} 8(1,4) \rightarrow 7(0,3)$ & 229758.7 & $10.5^{\prime \prime}$ & 0.42 & $(0,0)$ \\
\hline $\mathrm{CH}_{3} \mathrm{OH} 11(1,4) \rightarrow 10(2,4)$ & 104300.5 & $24.5^{\prime \prime}$ & 0.68 & $(0,0)$ \\
\hline $\mathrm{H}_{2} \mathrm{CO} 3_{12} \rightarrow 2_{11}$ & 225697.8 & $10.5^{\prime \prime}$ & 0.43 & $60^{\prime \prime} \times 60^{\prime \prime} \operatorname{map}(\mathrm{FIRS} 2), 72^{\prime \prime} \times 72^{\prime \prime} \operatorname{map}(\mathrm{LkH} \alpha 234)$ \\
\hline $\mathrm{H}_{2} \mathrm{CO} 2_{12} \rightarrow 1_{11}$ & 140839.5 & $18^{\prime \prime}$ & 0.57 & $60^{\prime \prime} \times 60^{\prime \prime} \operatorname{map}(\mathrm{FIRS} 2), 72^{\prime \prime} \times 72^{\prime \prime} \operatorname{map}(\mathrm{LkH} \alpha 234)$ \\
\hline $\mathrm{H}_{2}{ }^{13} \mathrm{CO} 2_{12} \rightarrow 1_{11}$ & 137449.9 & $18^{\prime \prime}$ & 0.57 & $(0,0)$ \\
\hline $\mathrm{CH}_{3} \mathrm{CN} 5(0) \rightarrow 4(0)$ & 91987.0 & $27^{\prime \prime}$ & 0.72 & $(0,0)$ \\
\hline $\mathrm{CH}_{3} \mathrm{CN} 5(1) \rightarrow 4(1)$ & 91985.3 & $27^{\prime \prime}$ & 0.72 & $(0,0)$ \\
\hline $\mathrm{CH}_{3} \mathrm{CN} 5(2) \rightarrow 4(2)$ & 91980.0 & $27^{\prime \prime}$ & 0.72 & $(0,0)$ \\
\hline $\mathrm{CH}_{3} \mathrm{CN} 5(3) \rightarrow 4(3)$ & 91971.4 & $27^{\prime \prime}$ & 0.72 & $(0,0)$ \\
\hline $\mathrm{CN} 2 \rightarrow 1$ & 226874.7 & $11^{\prime \prime}$ & 0.42 & $60^{\prime \prime} \times 60^{\prime \prime} \operatorname{map}(\mathrm{FIRS} 2), 60^{\prime \prime} \times 100^{\prime \prime}(\mathrm{LkH} \alpha 234)$ \\
\hline $\mathrm{N}_{2} \mathrm{D}^{+} 3 \rightarrow 2$ & 231321.7 & $10.5^{\prime \prime}$ & 0.42 & $(0,0)$ \\
\hline $\mathrm{DCO}^{+} 2 \rightarrow 1$ & 144077.3 & $17^{\prime \prime}$ & 0.56 & $60^{\prime \prime} \times 60^{\prime \prime}$ map \\
\hline $\mathrm{DCO}^{+} 3 \rightarrow 2$ & 216112.6 & $11^{\prime \prime}$ & 0.44 & $60^{\prime \prime} \times 60^{\prime \prime}$ map \\
\hline $\mathrm{D}_{2} \mathrm{CO} 4_{04} \rightarrow 3_{03}$ & 231410.3 & $10.5^{\prime \prime}$ & 0.42 & $(0,0)$ \\
\hline
\end{tabular}

${ }^{a}$ Only observed with a frequency resolution of $1 \mathrm{MHz}$. 
Table 2. Observational parameters towards NGC 7129 - FIRS 2.

\begin{tabular}{|c|c|c|c|c|}
\hline Line & $T_{\mathrm{MB}} \times \tau_{m}$ & $V\left(\mathrm{~km} \mathrm{~s}^{-1}\right)$ & $\Delta V\left(\mathrm{~km} \mathrm{~s}^{-1}\right)$ & $\overline{\tau_{m}}$ \\
\hline $\mathrm{NH}_{3}(1,1)$ & $3.08(0.07)$ & $-9.59(0.01)$ & $1.0(0.1)$ & $0.1^{a}$ \\
\hline $\mathrm{NH}_{3}(2,2)$ & $0.70(0.02)$ & $-9.63(0.02)$ & $1.3(0.1)$ & $0.1^{a}$ \\
\hline $\mathrm{NH}_{3}(3,3)$ & $0.12(0.01)$ & $-9.35(0.18)$ & $3.8(0.5)$ & $0.1^{a}$ \\
\hline $\mathrm{NH}_{3}(4,4)$ & \multicolumn{4}{|c|}{$\mathrm{rms}=0.05 \mathrm{~K} \mathrm{~km} \mathrm{~s}^{-1}$ with $\Delta V=3.0 \mathrm{~km} \mathrm{~s}^{-1}$} \\
\hline $\mathrm{N}_{2} \mathrm{H}^{+} 1 \rightarrow 0$ & $9.22(0.02)$ & $-9.51(0.01)$ & $1.91(0.01)$ & $1.28(0.10)$ \\
\hline $\mathrm{CN} 1 \rightarrow 0$ & $2.3(0.3)$ & $-9.7(0.1)$ & $1.4(0.1)$ & 1.9 \\
\hline $\mathrm{HCN} 1 \rightarrow 0$ & $2.3(0.3)$ & $-9.3(0.1)$ & $3.1(0.1)$ & 0.7 \\
\hline Line & Area $\left(\mathrm{K} \mathrm{km} \mathrm{s}^{-1}\right)$ & $V\left(\mathrm{~km} \mathrm{~s}^{-1}\right)$ & $\Delta V\left(\mathrm{~km} \mathrm{~s}^{-1}\right)$ & $T_{\mathrm{MB}}(\mathrm{K})$ \\
\hline $\mathrm{H}^{13} \mathrm{CO}^{+} 1 \rightarrow 0$ & $1.92(0.02)$ & $-9.74(0.01)$ & $1.27(0.02)$ & 1.39 \\
\hline $\mathrm{HC}^{18} \mathrm{O}^{+} 1 \rightarrow 0$ & $0.17(0.02)$ & $-9.68(0.09)$ & $1.1(0.2)$ & 0.14 \\
\hline \multirow[t]{2}{*}{$\mathrm{SiO} 2 \rightarrow 1$} & $1.55(0.09)$ & $-7.5(0.2)$ & $6.8(0.4)$ & 0.21 \\
\hline & $0.23(0.06)$ & $-9.5(0.1)$ & $1.6(0.4)$ & 0.13 \\
\hline $\mathrm{SiO} 3 \rightarrow 2$ & $2.50(0.20)$ & $-7.3(0.2)$ & $6.9(0.7)$ & 0.34 \\
\hline \multirow[t]{2}{*}{$\mathrm{CS} 3 \rightarrow 2$} & $3.69(0.12)$ & $-9.1(0.1)$ & $5.81(0.24)$ & 0.60 \\
\hline & $1.88(0.09)$ & $-9.9(0.1)$ & $1.19(0.04)$ & 1.48 \\
\hline \multirow[t]{2}{*}{$\mathrm{C}^{34} \mathrm{~S} 2 \rightarrow 1$} & $0.16(0.08)$ & $-7.8(0.8)$ & $3.1(1.3)$ & 0.05 \\
\hline & $0.28(0.07)$ & $-9.8(0.1)$ & $1.3(0.2)$ & 0.20 \\
\hline $\mathrm{C}^{34} \mathrm{~S} 3 \rightarrow 2$ & $1.05(0.08)$ & $-9.4(0.1)$ & $4.3(0.4)$ & 0.23 \\
\hline $\mathrm{C}^{34} \mathrm{~S} 5 \rightarrow 4$ & $1.21(0.16)$ & $-8.8(0.3)$ & $4.2(0.7)$ & 0.27 \\
\hline $\mathrm{CH}_{3} \mathrm{OH} 2(1,3) \rightarrow 1(1,3)$ & $0.6(0.3)$ & $-9(1)$ & $5(3)$ & 0.11 \\
\hline $\mathrm{CH}_{3} \mathrm{OH} 2(0,3) \rightarrow 1(0,3)$ & $1.85(0.06)$ & $-8.20(0.09)$ & $6.1(0.2)$ & 0.28 \\
\hline $\mathrm{CH}_{3} \mathrm{OH} 2(1,4) \rightarrow 1(1,4)$ & $4.71(0.05)$ & $-9.2(0.2)$ & $3.0(0.2)$ & 1.47 \\
\hline $\mathrm{CH}_{3} \mathrm{OH} 2(0,1) \rightarrow 1(0,1)$ & $5.83(0.07)$ & $-9.58(0.01)$ & $5.3(0.1)$ & 1.03 \\
\hline $\mathrm{CH}_{3} \mathrm{OH} 3(0,3) \rightarrow 2(0,3)$ & $4.12(0.04)$ & $-8.78(0.08)$ & $6.98(0.08)$ & 0.55 \\
\hline $\mathrm{CH}_{3} \mathrm{OH} 3(1,4) \rightarrow 2(1,4)$ & $8.9(0.09)$ & $-8.32(0.01)$ & $5.71(0.07)$ & 1.46 \\
\hline $\mathrm{CH}_{3} \mathrm{OH} 3(0,1) \rightarrow 2(0,1)$ & $9.6(0.1)$ & $-8.34(0.02)$ & $5.07(0.06)$ & 1.78 \\
\hline $\mathrm{CH}_{3} \mathrm{OH} 3(2,2) \rightarrow 2(2,2)$ & $2(1)$ & $-12(4)$ & $7(10)$ & 0.27 \\
\hline $\mathrm{CH}_{3} \mathrm{OH} 3(1,3) \rightarrow 2(1,3)$ & $1.9(0.1)$ & $-8.7(0.2)$ & $6.4(0.5)$ & 0.28 \\
\hline $\mathrm{CH}_{3} \mathrm{OH} 5(2,4) \rightarrow 4(2,4)$ & $3(1)$ & $-8.9(0.7)$ & $4(2)$ & 0.70 \\
\hline $\mathrm{CH}_{3} \mathrm{OH} 5(0,1) \rightarrow 4(0,1)$ & $12.3(0.7)$ & $-7.4(0.1)$ & $4.6(0.3)$ & 2.51 \\
\hline $\mathrm{CH}_{3} \mathrm{OH} 5(1,4) \rightarrow 4(1,4)$ & $8.8(0.8)$ & $-7.8(0.2)$ & $4.6(0.5)$ & 1.80 \\
\hline $\mathrm{CH}_{3} \mathrm{OH} 5(3,1) \rightarrow 4(3,1)$ & $1.7(1.7)$ & $-8.1(3)$ & $6.9(7)$ & 0.23 \\
\hline $\mathrm{CH}_{3} \mathrm{OH} 5(2,2) \rightarrow 4(2,2)$ & $1.3(1.5)$ & $-10(4)$ & $7(11)$ & 0.17 \\
\hline $\mathrm{CH}_{3} \mathrm{OH} 8(1,4) \rightarrow 7(0,3)$ & $4.5(0.4)$ & $-7.7(0.1)$ & $4.3(0.3)$ & 0.98 \\
\hline $\mathrm{CH}_{3} \mathrm{OH} 11(1,4) \rightarrow 10(2,4)$ & $0.14(0.04)$ & $-9.9(0.4)$ & $3.6(5.9)$ & 0.04 \\
\hline \multirow[t]{2}{*}{$\mathrm{H}_{2} \mathrm{CO} 3_{12} \rightarrow 2_{11}$} & $2.3(0.2)$ & $-9.6(0.1)$ & $1.3(0.1)$ & 1.66 \\
\hline & $6.7(0.3)$ & $-8.6(0.2)$ & $7.7(0.5)$ & 0.81 \\
\hline \multirow[t]{2}{*}{$\mathrm{H}_{2} \mathrm{CO} 2_{12} \rightarrow 1_{11}$} & $3.0(0.1)$ & $-9.66(0.01)$ & $1.41(0.03)$ & 2.00 \\
\hline & $6.0(0.1)$ & $-8.97(0.06)$ & $6.3(0.2)$ & 0.91 \\
\hline $\mathrm{H}_{2}{ }^{13} \mathrm{CO} 2_{12} \rightarrow 1_{11}$ & $0.33(0.04)$ & $-9.4(0.2)$ & $2.8(0.5)$ & 0.11 \\
\hline $\mathrm{CH}_{3} \mathrm{CN} 5(0) \rightarrow 4(0)$ & $0.24(0.05)$ & $-9.1(0.4)$ & $4.2(0.8)$ & 0.05 \\
\hline $\mathrm{CH}_{3} \mathrm{CN} 5(1) \rightarrow 4(1)$ & $0.25(0.05)$ & $-9.1(0.6)$ & $5(1)$ & 0.05 \\
\hline $\mathrm{CH}_{3} \mathrm{CN} 5(2) \rightarrow 4(2)$ & $0.11(0.02)$ & $-9.7(0.9)$ & $4(2)$ & 0.02 \\
\hline $\mathrm{CH}_{3} \mathrm{CN} 5(3) \rightarrow 4(3)$ & $0.12(0.02)$ & $-8(1)$ & $7(3)$ & 0.02 \\
\hline $\mathrm{CN} 2 \rightarrow 1$ & $2.74(0.24)$ & $-9.7(0.1)$ & $2.6(0.2)$ & 1.0 \\
\hline $\mathrm{N}_{2} \mathrm{D}^{+} 3 \rightarrow 2$ & $0.64(0.05)$ & $-9.90(0.04)$ & $1.1(0.1)$ & 0.53 \\
\hline $\mathrm{DCO}^{+} 2 \rightarrow 1$ & $2.93(0.04)$ & $-9.86(0.01)$ & $1.0(0.1)$ & 2.61 \\
\hline $\mathrm{DCO}^{+} 3 \rightarrow 2$ & $1.9(0.1)$ & $-9.73(0.02)$ & $1.0(0.1)$ & 1.83 \\
\hline $\mathrm{D}_{2} \mathrm{CO} 4_{04} \rightarrow 3_{03}$ & $0.3(0.1)$ & $-9.6(0.3)$ & $3.9(1)$ & 0.05 \\
\hline
\end{tabular}

${ }^{a}$ In case of optically thin emission, $\tau_{m}$ cannot be determined and is set arbitrarily to 0.1 . 
Table 3. Observational parameters towards $\mathrm{LkH} \alpha 234$

\begin{tabular}{|c|c|c|c|c|}
\hline Line & $\overline{T_{\mathrm{MB}} \times \tau_{m}}$ & $\bar{V}\left(\mathrm{~km} \mathrm{~s}^{-1}\right)$ & $\Delta V\left(\mathrm{~km} \mathrm{~s}^{-1}\right)$ & $\bar{\tau} \tau_{m}$ \\
\hline $\mathrm{NH}_{3}(1,1)$ & $0.60(0.03)$ & $-9.87(0.02)$ & $0.9(0.2)$ & $0.86(0.07)$ \\
\hline $\mathrm{NH}_{3}(2,2)$ & $0.27(0.04)$ & $-9.94(0.05)$ & $1.1(0.2)$ & $0.1^{a}$ \\
\hline $\mathrm{NH}_{3}(3,3)$ & $0.10(0.03)$ & $-11(1)$ & $1.6(0.7)$ & $0.1^{a}$ \\
\hline $\mathrm{NH}_{3}(4,4)$ & \multicolumn{4}{|c|}{$\mathrm{rms}=0.13 \mathrm{~K} \mathrm{~km} \mathrm{~s}^{-1}$ with $\Delta V=1.1 \mathrm{~km} \mathrm{~s}^{-1}$} \\
\hline $\mathrm{N}_{2} \mathrm{H}^{+} 1 \rightarrow 0$ & $1.53(0.06)$ & $-9.48(0.01)$ & $1.82(0.04)$ & $1.32(0.7)$ \\
\hline $\mathrm{CN} 1 \rightarrow 0$ & $1.9(0.2)$ & $-9.9(0.1)$ & $2.6(0.1)$ & 0.8 \\
\hline $\mathrm{HCN} 1 \rightarrow 0$ & $3.2(0.3)$ & $-10.0(0.1)$ & $2.9(0.1)$ & $0.1^{a}$ \\
\hline Line & Area $\left(\mathrm{K} \mathrm{km} \mathrm{s}^{-1}\right)$ & $V\left(\mathrm{~km} \mathrm{~s}^{-1}\right)$ & $\Delta V\left(\mathrm{~km} \mathrm{~s}^{-1}\right)$ & $T_{\mathrm{MB}}(\mathrm{K})$ \\
\hline $\mathrm{H}^{13} \mathrm{CO}^{+} 1 \rightarrow 0$ & $1.00(0.03)$ & $-9.80(0.03)$ & $1.79(0.07)$ & 0.52 \\
\hline $\mathrm{HC}^{18} \mathrm{O}^{+} 1 \rightarrow 0$ & $0.14(0.04)$ & $-10.1(0.2)$ & $2(1)$ & 0.06 \\
\hline $\mathrm{SiO} 2 \rightarrow 1$ & $0.31(0.06)$ & $-9.9(0.9)$ & $8.5(2.0)$ & 0.03 \\
\hline $\mathrm{SiO} 3 \rightarrow 2$ & $0.60(0.08)$ & $-8.7(0.2)$ & $3.2(0.5)$ & 0.18 \\
\hline $\mathrm{CS} 3 \rightarrow 2$ & $14.9(0.2)$ & $-9.7(0.1)$ & $3.0(0.1)$ & 4.67 \\
\hline $\mathrm{C}^{34} \mathrm{~S} 2 \rightarrow 1$ & $0.66(0.04)$ & $-9.9(0.1)$ & $3.4(0.2)$ & 0.18 \\
\hline $\mathrm{C}^{34} \mathrm{~S} 3 \rightarrow 2$ & $1.41(0.05)$ & $-9.5(0.1)$ & $3.2(0.2)$ & 0.41 \\
\hline$C^{34} S 5 \rightarrow 4$ & $1.71(0.22)$ & $-9.1(0.3)$ & $4.5(0.8)$ & 0.36 \\
\hline $\mathrm{CH}_{3} \mathrm{OH} 2(1,3) \rightarrow 1(1,3)$ & $0.3(0.1)$ & $-7(3)$ & $12(5)$ & 0.02 \\
\hline $\mathrm{CH}_{3} \mathrm{OH} 2(0,3) \rightarrow 1(0,3)$ & $0.20(0.04)$ & $-9.8(0.3)$ & $2.9(0.7)$ & 0.06 \\
\hline $\mathrm{CH}_{3} \mathrm{OH} 2(1,4) \rightarrow 1(1,4)$ & $0.48(0.03)$ & $-9.6(0.1)$ & $2.2(0.2)$ & 0.20 \\
\hline $\mathrm{CH}_{3} \mathrm{OH} 2(0,1) \rightarrow 1(0,1)$ & $0.64(0.04)$ & $-9.5(0.1)$ & $5.2(0.4)$ & 0.11 \\
\hline $\mathrm{CH}_{3} \mathrm{OH} 3(0,3) \rightarrow 2(0,3)$ & $1.27(0.09)$ & $-9.5(0.1)$ & $4.6(0.4)$ & 0.26 \\
\hline $\mathrm{CH}_{3} \mathrm{OH} 3(1,4) \rightarrow 2(1,4)$ & $1.91(0.09)$ & $-9.2(0.1)$ & $4.8(0.3)$ & 0.37 \\
\hline $\mathrm{CH}_{3} \mathrm{OH} 3(0,1) \rightarrow 2(0,1)$ & $1.94(0.07)$ & $-9.2(0.1)$ & $3.3(0.2)$ & 0.55 \\
\hline $\mathrm{CH}_{3} \mathrm{OH} 3(2,2) \rightarrow 2(2,2)$ & $1.6(0.4)$ & $-12(1)$ & $9(4)$ & 0.17 \\
\hline $\mathrm{CH}_{3} \mathrm{OH} 3(1,3) \rightarrow 2(1,3)$ & $1.3(0.4)$ & $-10(1)$ & $8(3)$ & 0.15 \\
\hline $\mathrm{CH}_{3} \mathrm{OH} 5(2,4) \rightarrow 4(2,4)$ & $2.6(0.5)$ & $-9.4(0.5)$ & $5(1)$ & 0.49 \\
\hline $\mathrm{CH}_{3} \mathrm{OH} 5(0,1) \rightarrow 4(0,1)$ & $2.9(0.2)$ & $-8.8(0.1)$ & $4.1(0.6)$ & 0.66 \\
\hline $\mathrm{CH}_{3} \mathrm{OH} 5(1,4) \rightarrow 4(1,4)$ & $2.1(0.2)$ & $-9.2(0.1)$ & $3.1(0.2)$ & 0.64 \\
\hline $\mathrm{CH}_{3} \mathrm{OH} 8(1,4) \rightarrow 7(0,3)$ & $2.0(0.3)$ & $-9.7(0.5)$ & $7(1)$ & 0.27 \\
\hline $\mathrm{CH}_{3} \mathrm{OH} 11(1,4) \rightarrow 10(2,4)$ & $0.13(0.04)$ & $-9.9(0.8)$ & $6(2)$ & 0.02 \\
\hline \multirow[t]{2}{*}{$\mathrm{H}_{2} \mathrm{CO} 3_{12} \rightarrow 2_{11}$} & $5.4(0.3)$ & $-9.4(0.1)$ & $2.2(0.1)$ & 2.2 \\
\hline & $5.9(0.3)$ & $-9.8(0.1)$ & $5.5(0.2)$ & 1.0 \\
\hline \multirow[t]{2}{*}{$\mathrm{H}_{2} \mathrm{CO} 2_{12} \rightarrow 1_{11}$} & $8.1(0.6)$ & $-9.7(0.1)$ & $2.4(0.1)$ & 3.1 \\
\hline & $3.1(0.6)$ & $-9.5(0.2)$ & $6(1)$ & 0.5 \\
\hline $\mathrm{H}_{2}{ }^{13} \mathrm{CO} 2_{12} \rightarrow 1_{11}$ & $0.24(0.05)$ & $-9.4(0.4)$ & $4(1)$ & 0.05 \\
\hline $\mathrm{CH}_{3} \mathrm{CN} 5(0) \rightarrow 4(0)$ & $0.17(0.02)$ & $-10.2(0.2)$ & $2.6(0.4)$ & 0.06 \\
\hline $\mathrm{CH}_{3} \mathrm{CN} 5(1) \rightarrow 4(1)$ & $0.16(0.02)$ & $-9.9(0.2)$ & $2.9(0.4)$ & 0.05 \\
\hline $\mathrm{CH}_{3} \mathrm{CN} 5(2) \rightarrow 4(2)$ & \multicolumn{4}{|c|}{$<0.07 \mathrm{~K} \mathrm{~km} \mathrm{~s}^{-1}$ with $\Delta V=3.2 \mathrm{~km} \mathrm{~s}^{-1}$} \\
\hline $\mathrm{CH}_{3} \mathrm{CN} 5(3) \rightarrow 4(3)$ & \multicolumn{4}{|c|}{$<0.07 \mathrm{~K} \mathrm{~km} \mathrm{~s}^{-1}$ with $\Delta V=3.2 \mathrm{~km} \mathrm{~s}^{-1}$} \\
\hline $\mathrm{CN} 2 \rightarrow 1$ & $6.0(0.3)$ & $-9.0(0.1)$ & $2.9(0.2)$ & 1.9 \\
\hline $\mathrm{N}_{2} \mathrm{D}^{+} 3 \rightarrow 2$ & \multicolumn{4}{|c|}{$\sigma=0.11 \mathrm{~K} \mathrm{~km} \mathrm{~s}^{-1}$ en $\Delta V=1.5 \mathrm{~km} \mathrm{~s}^{-1}$} \\
\hline $\mathrm{DCO}^{+} 2 \rightarrow 1$ & $0.62(0.04)$ & $-9.58(0.04)$ & $1.5(0.1)$ & 0.38 \\
\hline $\mathrm{DCO}^{+} 3 \rightarrow 2$ & $0.76(0.07)$ & $-9.4(0.1)$ & $2.2(0.2)$ & 0.33 \\
\hline $\mathrm{D}_{2} \mathrm{CO} 4_{04} \rightarrow 3_{03}$ & $0.60(0.2)$ & $-9.1(0.5)$ & $4(1)$ & 0.15 \\
\hline
\end{tabular}

${ }^{a}$ In case of optically thin emission, $\tau_{m}$ cannot be determined and is set arbitrarily to 0.1 . 